\title{
A Review on Computer Aided Diagnosis of Acute Brain Stroke
}

\author{
Mahesh Anil Inamdar ${ }^{1}$ (D), Udupi Raghavendra 2,*iD, Anjan Gudigar ${ }^{2}$, Yashas Chakole ${ }^{2}$, Ajay Hegde ${ }^{3}$ (D), \\ Girish R. Menon ${ }^{3}$, Prabal Barua ${ }^{4,5,6}{ }^{(D)}$, Elizabeth Emma Palmer ${ }^{7}$, Kang Hao Cheong ${ }^{8}$ (D), Wai Yee Chan ${ }^{9}$, \\ Edward J. Ciaccio ${ }^{10}$ and U. Rajendra Acharya $11,12,13,14$ (D)
}

1 Department of Mechatronics, Manipal Institute of Technology, Manipal Academy of Higher Education, Manipal 576104, India; mahesh.inamdar@manipal.edu

2 Department of Instrumentation and Control Engineering, Manipal Institute of Technology, Manipal Academy of Higher Education, Manipal 576104, India; anjan.gudigar@manipal.edu (A.G.); yashaschakole1999@gmail.com (Y.C.)

3 Department of Neurosurgery, Kasturba Medical College, Manipal Academy of Higher Education, Manipal 576104, India; dr.ajayhegde@gmail.com (A.H.); girish.menon@manipal.edu (G.R.M.)

4 School of Management \& Enterprise, University of Southern Queensland, Toowoomba, QLD 4350, Australia; prabal.barua@usq.edu.au

5 Faculty of Engineering and Information Technology, University of Technology, Sydney, NSW 2007, Australia

6 Cogninet Brain Team, Cogninet Australia, Sydney, NSW 2010, Australia

7 School of Women's and Children's Health, University of New South Wales, Sydney, NSW 2052, Australia; elizabeth.palmer@health.nsw.gov.au

8 Science, Mathematics and Technology Cluster, Singapore University of Technology and Design, Singapore 487372, Singapore; kanghao_cheong@sutd.edu.sg

check for updates

Citation: Inamdar, M.A.; Raghavendra, U.; Gudigar, A.; Chakole, Y.; Hegde, A.; Menon, G.R.; Barua, P.; Palmer, E.E.; Cheong, K.H.; Chan, W.Y.; et al. A Review on Computer Aided Diagnosis of Acute Brain Stroke. Sensors 2021, 21, 8507. https://doi.org/10.3390/s21248507

Academic Editors: Ewa Pietka, Arkadiusz Gertych and Wojciech Więcławek

Received: 4 October 2021

Accepted: 9 December 2021

Published: 20 December 2021

Publisher's Note: MDPI stays neutral with regard to jurisdictional claims in published maps and institutional affiliations.

Copyright: (c) 2021 by the authors. Licensee MDPI, Basel, Switzerland. This article is an open access article distributed under the terms and conditions of the Creative Commons Attribution (CC BY) license (https:/ / creativecommons.org/licenses/by/ $4.0 /)$.
9 Department of Biomedical Imaging, Research Imaging Centre, University of Malaya, Kuala Lumpur 59100, Malaysia; waiyeec@ummc.edu.my

10 Department of Medicine, Columbia University, New York, NY 10032, USA; ciaccio@columbia.edu

11 Department of Biomedical Engineering, Faculty of Engineering, University of Malaya, Kuala Lumpur 50603, Malaysia; aru@np.edu.sg

12 School of Engineering, Ngee Ann Polytechnic, Singapore 599489, Singapore

13 Department of Biomedical Engineering, School of Science and Technology, SUSS University, Singapore 599491, Singapore

14 Department of Biomedical Informatics and Medical Engineering, Asia University, Taichung 41354, Taiwan

* Correspondence: raghavendra.u@manipal.edu

\begin{abstract}
Amongst the most common causes of death globally, stroke is one of top three affecting over 100 million people worldwide annually. There are two classes of stroke, namely ischemic stroke (due to impairment of blood supply, accounting for $~ 70 \%$ of all strokes) and hemorrhagic stroke (due to bleeding), both of which can result, if untreated, in permanently damaged brain tissue. The discovery that the affected brain tissue (i.e., 'ischemic penumbra') can be salvaged from permanent damage and the bourgeoning growth in computer aided diagnosis has led to major advances in stroke management. Abiding to the Preferred Reporting Items for Systematic Review and Meta-Analyses (PRISMA) guidelines, we have surveyed a total of 177 research papers published between 2010 and 2021 to highlight the current status and challenges faced by computer aided diagnosis (CAD), machine learning (ML) and deep learning (DL) based techniques for CT and MRI as prime modalities for stroke detection and lesion region segmentation. This work concludes by showcasing the current requirement of this domain, the preferred modality, and prospective research areas.
\end{abstract}

Keywords: Ischemic brain stroke; machine learning; deep learning; CAD

\section{Introduction}

In the recent past, stroke has become the foremost cause of mortality and healthdisability worldwide, causing over 6.6 million deaths annually [1], and with up to $50 \%$ of survivors being chronically disabled [2]. Economic impacts post-treatment and for poststroke care are substantial [1,2]. Risk factors for stroke are both non-modifiable/inherent 
and modifiable. Former risk factors include age, gender and genetic factors: with stroke incidence being higher in older individuals and men [3]. Hypertension, smoking, high alcohol consumption, waist-to-hip ratio, and diet are amongst the modifiable risk factor for stroke, particularly ischemic stroke [4].

Brain strokes are of two types: ischemic (due to intracerebral vessel occlusion) and hemorrhagic (due to intracerebral bleeding), as depicted in Figure 1.

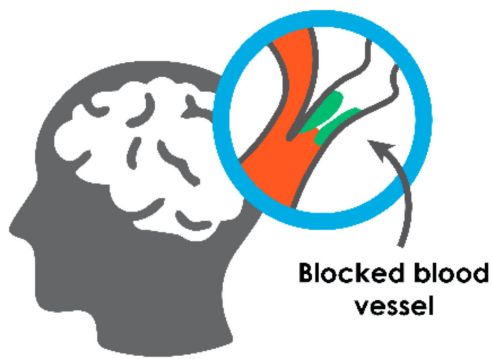

Ischemic Stroke

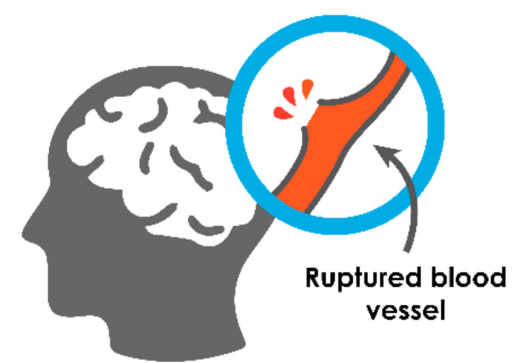

Haemorrhagic Stroke

Figure 1. Ischemic and hemorrhagic brain stroke.

Ischemic strokes are globally more common, accounting for at least $70 \%$ of all strokes. It is most often caused by thrombosis (clots) secondary to large artery atherosclerosis, or emboli from the heart in cardiac disease, such as atrial fibrillation [5]. Other causes of ischemic stroke are small vessel disease, arterial dissections (tears), vasculitis, hypotensive vasoconstriction [5], and hematological disorders (for example, sickle cell anemia, which is a leading cause of pediatric stroke in Africa) [6]. Epidemiological patterns vary between countries. The reductions in incidence, mortality, and disability-adjusted life years in ischemic strokes in high-income countries, largely attributed to improved lifestyles and rapid detection and treatments, has not yet been seen in low and middle-income countries [7]. This widening health inequity is a major challenge, which needs to be urgently tackled, and more emphasis must to be given to democratize cost effective AI solutions for diagnosis and stratifications. In India, the largest contributors to total neurological disorder were strokes (37.9\%), causing $7.4 \%$ of total deaths [8]. Stratification by gender for the Indian population is shown in Figure 2.

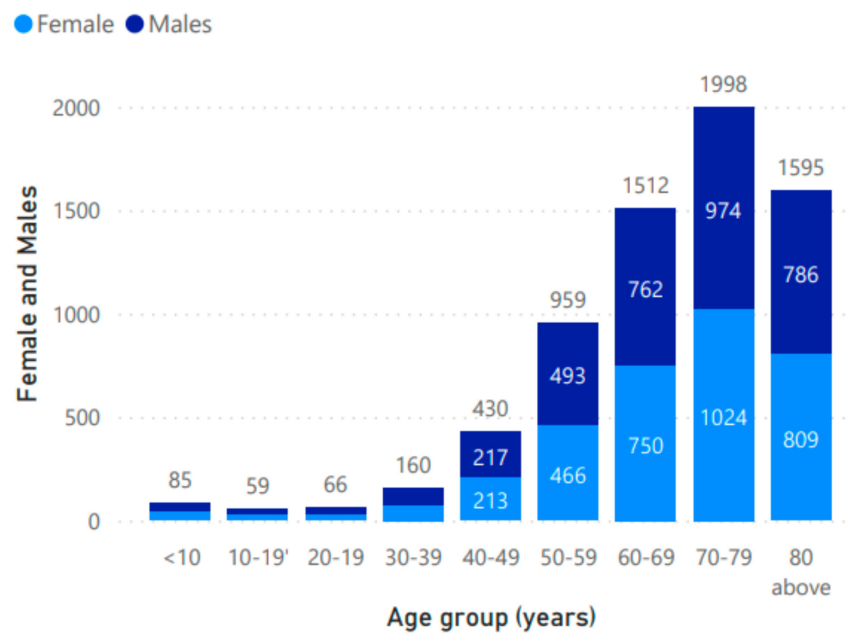

Figure 2. Age-specific incidence rate of strokes by gender in India, 2019.

Although hemorrhagic strokes account for $10-15 \%$ of total stroke incidence, they are associated with very high mortality and morbidity, which has not improved globally over the last 20 years. Mortality is $>50 \%$ with half of the deaths occurring in the first two days. 
Only $20 \%$ of patients manage to gain independent activity daily living (ADL) six months after a hemorrhagic stroke. Uncontrolled hypertension and vascular malfunction are the leading causes of hemorrhagic stokes $[9,10]$.

Since the 1970s, it has been recognized that following an acute stroke there are hypoperfused brain regions, the 'penumbral regions', which are supplied by alternative blood flow pathways to the one which is occluded. These penumbral regions can potentially be rescued from permanent cell death if identified in time for reperfusion therapy (restoration of normal blood supply), as well as treatments for the underlying cause of an individual's stroke [11]. For ischemic stroke due to thromboembolism (the most common cause of strokes) this would include intravenous thrombolysis medication therapy. The time window for a successful and most effective treatment is very small [12]. For example, best outcomes for the thrombolytic medication alteplase are achieved when this is started within $3 \mathrm{~h}$ [13]. This ultra-rapid, tailored management approach, ideally within dedicated stroke units, is a key recommendation of the World Stroke Organization's global guidelines, and essential in reducing mortality and morbidity [14].

However, intravenous thrombolysis is contraindicated if there is presence of hemorrhage in the infarcted area. Therefore, stroke guidelines recommend that all patients who are candidates for reperfusion therapies should undergo immediate neuroimaging, ideally with MRI, and all other suspected stroke patients should have an urgent brain computed tomogram (CT) or magnetic resonance imaging (MRI), preferably within $60 \mathrm{~min}$ [15]. Moreover, if reperfusion therapy has been escalated, repeat neuroimaging, approximately $24 \mathrm{~h}$ after therapy administration, is recommended to identify early complication such as hemorrhagic transformation, and to reassess the infarcted core. This is crucial to decide the next appropriate treatments (for example, anti-thrombotic therapies and deep vein thrombosis prophylaxis) [16].

Thus, accurate interpretation of neuroimaging to guide most appropriate treatment decisions ('image-based treatment guidance') is a key part of modern optimal stroke management. This can be very challenging, and dependent on highly skilled radiologists working under intense time pressures, who are able to carefully analyze a very large amount of radiological data to accurately identify normal and infarcted regions ('lesion segmentation'), as well as underlying pathophysiological mechanisms such as arterial occlusions. The optimal approach to neuroimaging analysis has been intensively studied, and the possibility of incorporation of artificial intelligence, machine learning (ML), and deep learning (DL), has been increasingly explored [17]. Figure 3 shows the current role AI plays in the brain stroke process.

\section{ROLES OF ARTIFICIAL INTELLIGENCE IN BRAIN STROKE MANAGEMENT PIPELINE}

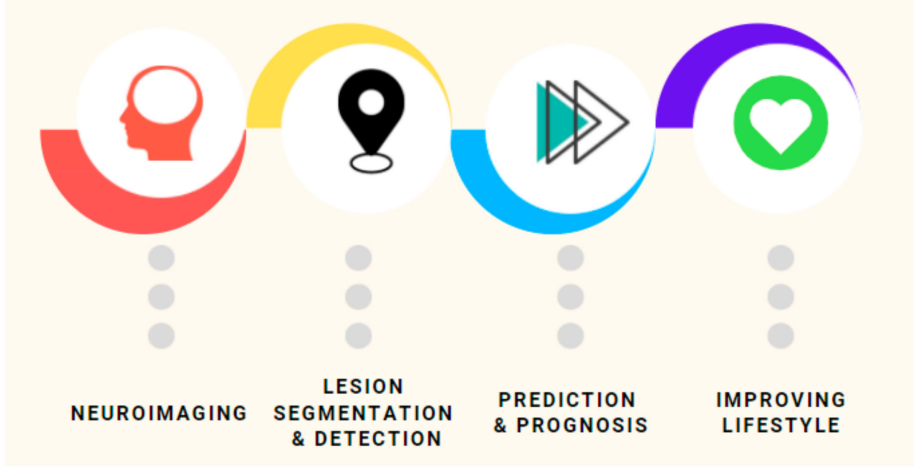

Figure 3. Schematic to showcase applications of AI in stroke management.

Both CT and MRI are used in stroke management globally [18]. A more time-saving imaging modality is CT, and this is usually the first line of imaging. MRIs, on the other 
hand, employ electromagnetic waves to produce superior image resolution of the brain. An MRI with diffusion weighted sequence (DWI) is able to identify hyper-acute stroke events as early as within minutes of onset [19]. Thus, although CT scanners are more accessible globally, less expensive and quicker than MRIs, MRIs are more sensitive to detect acute ischemic events, and thus recommended (especially when there is a potential for reperfusion therapy) $[20,21]$. However, recent developments in CT technology, such as perfusion CT (CTP) to identify ischemic penumbra combined with $\mathrm{CT}$ angiography (CTA) to identify occluded vessels, represent promising alternatives to allow fast response and associated assessment of stroke patients in cases where MRI machines are either too expensive, inaccessible, or contraindicated [22].

The review articles in the literature have extensively investigated and discussed the recent advancements in neuroimaging, various techniques employed for detection and lesion segmentation (lesion stage wise by Yue), and the challenges involved in these techniques [23-27]. Although these studies have contributed tremendously, there is still scope for a deeper and more exhaustive study particularly based on modalities and their suitability to the current conditions. Moreover, we have identified that there was a need for a single comprehensive work amalgamating details of the recent development in all areas of this domain.

\subsection{Review Objective}

The main objective of this paper, besides discussing the most recent developments in diagnostic techniques, is to provide the reader with:

- A comprehensive overview of various modalities involved in neuroimaging, their characteristics, and requirement. We compare the most prominent ones and make remarks on their suitability, accessibility and viability. This will be useful in prioritizing future research avenues;

- An all-inclusive overview of a host of recent techniques (with special focus on prognosis) for stroke classification, detection and lesion segmentation categorized on the basis of modality used, techniques employed, datasets used (with benchmarks) (see Table 1) and the challenges faced;

- The areas of plausible future research. 
Table 1. Datasets and Benchmarks.

\begin{tabular}{|c|c|c|c|c|c|c|}
\hline Modality & Database & Data Size & Area & Classes & Ground Truth & Data Info \\
\hline MRI & ISLES 2015 & $\begin{array}{l}\text { SISS: } 28 \text { (train) } \\
\text { 36(test) } \\
\text { SPES: 30(train) } \\
\text { 20(test) }\end{array}$ & $\begin{array}{l}\text { SISS: sub-acute } \\
\text { ischemic stroke lesion } \\
\text { segmentation } \\
\text { SPES: acute stroke } \\
\text { outcome/penumbra } \\
\text { estimation }\end{array}$ & $\begin{array}{l}\text { SISS: Lesions were classified } \\
\text { as sub-acute infarct and } \\
\text { Infarct lesions. } \\
\text { SPES: target mismatch = } \\
\text { perfusion-restriction label } \\
\text { minus diffusion-restriction } \\
\text { label }\end{array}$ & $\begin{array}{l}\text { SISS: Segmentation by } \\
\text { an Expert }\end{array}$ & $\begin{array}{l}\text { http:/ / www.isles-challenge.org/ISLES2015 } \\
\text { (accessed on } 4 \text { October 2021). }\end{array}$ \\
\hline MRI & ISLES 2016 & 35 (train) 19(test) & $\begin{array}{l}\text { Dataset provides a } \\
\text { regression and } \\
\text { segmentation and a task: } \\
\text { Task 1: prediction of } \\
\text { Lesion outcome } \\
\text { Task 2: prediction of } \\
\text { Clinical outcome }\end{array}$ & 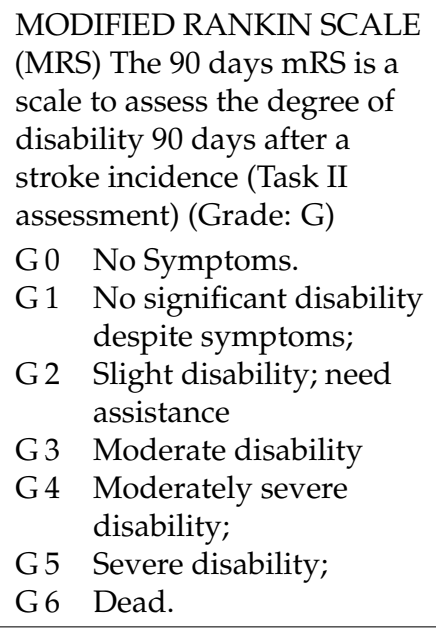 & $\begin{array}{l}\text { Final lesion volume } \\
\text { (Task } 1 \text { ) as manually and } \\
\text { the clinical mRM score } \\
\text { (Task } 2 \text { ) denoting the } \\
\text { extent of disability }\end{array}$ & $\begin{array}{c}\text { http:/ / www.isles-challenge.org/ISLES2016/ } \\
\text { Highest } \mathbf{I}_{\mathrm{DC}}=\mathbf{3 . 3 7}\end{array}$ \\
\hline $\begin{array}{l}\text { MRI- (DWI, } \\
\text { ADC) }\end{array}$ & ISLES 2017 & 43(train) 32(test) & $\begin{array}{l}\text { Acute ischemic stroke } \\
\text { (Challenge for stroke } \\
\text { lesions segmentation, } \\
\text { core and penumbra } \\
\text { separation) }\end{array}$ & & $\begin{array}{l}\text { Ground-truth } \\
\text { segmentation maps } \\
\text { manually drawn on } \\
\text { scans }\end{array}$ & $\begin{array}{l}\text { Lesion outcome (prediction) based on acute } \\
\text { MRI data. } \\
\text { http:/ / www.isles-challenge.org/ISLES2017/ } \\
\text { Highest } \mathbf{I}_{\mathrm{DC}}=\mathbf{4 . 5 3}\end{array}$ \\
\hline $\begin{array}{l}\text { CBF, MTT, } \\
\text { CBV, TMAX, } \\
\text { CTP }\end{array}$ & ISLES 2018 & 63 (train) 40(test) & $\begin{array}{l}\text { Penumbra-core } \\
\text { separation using CT }\end{array}$ & & $\begin{array}{l}\text { Expert segmentations of } \\
\text { the infarct lesions. }\end{array}$ & $\begin{array}{l}\text { Acute ischemic stroke patients with } 8 \text { hrs. of } \\
\text { stroke onset and MRI DWI within } 3 \mathrm{~h} \text {. after } \\
\text { CTP. http:/ / www.isles-challenge.org/ }\end{array}$ \\
\hline
\end{tabular}




\subsection{Article Search}

A systematic literature review was performed following PRISMA guidelines. A comprehensive database search was conducted to identify peer-reviewed articles published between 2010 and 2021 including the following terms 'Ischemic Stroke', 'Hemorrhagic Stroke', 'Lesion Segmentation', 'Prognosis of Brain Strokes', 'Lesion Detection and Segmentation', 'Penumbra Core' and 'Neuroimaging'. Search engines used were: Science Direct, IEEE Xplore, Spice, Springer and Wiley.

\subsection{Selection of Articles}

Studies published between 2010 and 2021 which strictly adhere to the subject area and a few earlier survey methods, case definitions and concept studies were considered. The entire process was carried out with three level filtering. 676 articles were initially collected and 322 publications were filtered out as they were not relevant to the subject and domain. A further 121 were filtered out due to the type of methods employed. 177 were finally shortlisted for analysis based on relevance, type of publication, modality and implementation details of technical aspects. Figure 4 shows articles selection process. To the best of our knowledge, we have gathered all publications between 2010 and 2021 covering this subject.

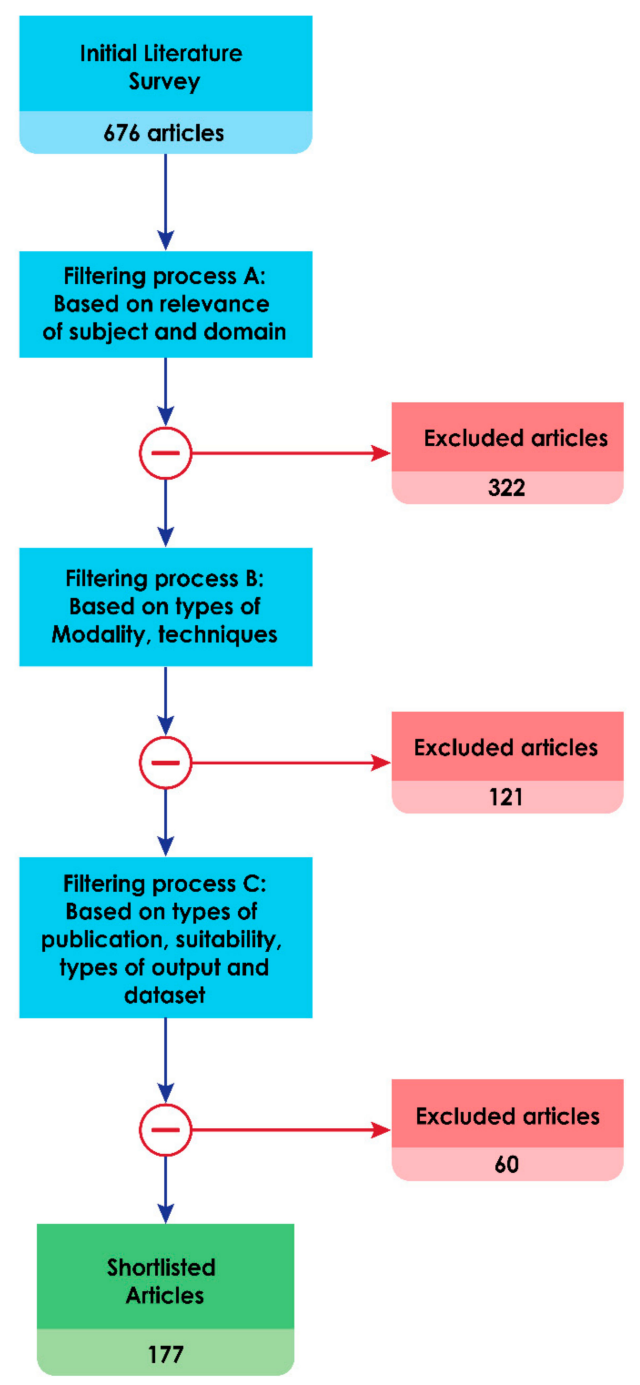

Figure 4. Articles selection process based on the PRISMA guidelines.

This systematic review has been written following the PRISMA guidelines. The inclusion and exclusion criteria are mentioned in Table 2. 
Table 2. The exclusion and inclusion criteria.

\begin{tabular}{ll}
\hline Inclusion & Exclusion \\
\hline Studies pertaining to & Studies pertaining to \\
1. CT and MRI (including variants) & 1. Treatment of strokes (Exclusively) \\
2. Ischemic and hemorrhagic strokes & 2. Pure Statistical and Biological methods of treatment. \\
3. Measurement of the degree of the infarct and damage. & 3. Technical working and advancement of algorithms \\
4. Prognosis of strokes and the likelihood of damage & 4. Lesions extraneous to strokes \\
5. Lesion detection and segmentation (core and penumbra region) & \\
6. ML and DL techniques for segmentation of lesion regions & \\
7. Latest architectures in DL techniques and factorization & \\
techniques for feature-specific algorithms. & \\
\hline
\end{tabular}

\subsection{Analysis of Articles}

177 papers which met the inclusion and exclusion criteria were analyzed and stratified based on imaging modality, techniques applied (ML/DL), and the types of problem addressed.

\subsection{Paper Structure}

Here, we mention the structure of the paper. In Section 2, a brief overview is provided of the basics of imaging in the brain stroke domain. It begins by describing the considered modalities with their description and working principles. Section 4 contains the prospects of deep learning and a comprehensive review of various techniques/architectures for stroke (ischemic/hemorrhagic) detection and lesion region segmentation and prognosis. In Section 5 we discuss learning, research gaps, and future scope. Finally, Section 6 contains the compendium of the research work. Figure 5 shows the structure of this paper.

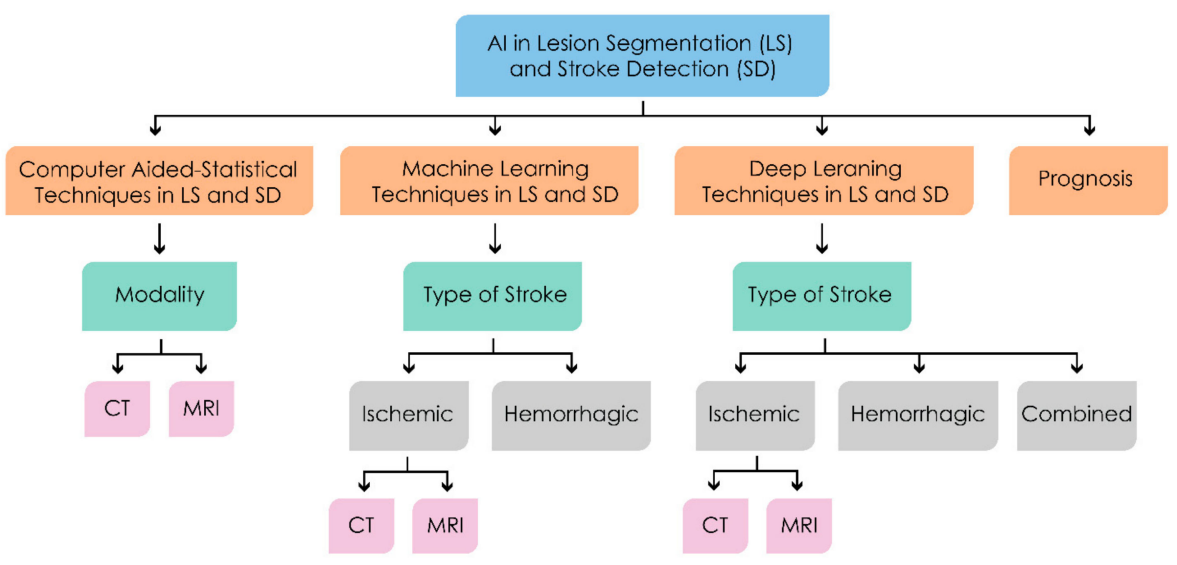

Figure 5. Structure of the review process.

\section{Brief Perspective on Brain Stroke Imaging}

Neurological abnormalities are captured through CT and MRI. CT perfusion imaging has been used to assess the degree of the ischemic penumbra, infarcted core and to aid treatment decision. CT in the acute setting is mainly to identify contraindication to thrombolysis and to exclude stroke mimics. In this section we present a brief overview of stroke imaging techniques and the modalities employed for diagnosis and treatment. Table 3 and Figure 5 provide detailed description of the considered modalities.

\subsection{Ischemic Stroke}

Reduced cerebral blood flow (CBF) due to the occlusion of blood vessels lead to ischemic stroke. Although ischemia tolerance differs between tissue types, these types of strokes can be fatal when there is large vessel occlusion [28]. The deprived tissue begins losing essential nutrients and oxygen and excretes toxins which accumulate and impact normal function. Failure of recanalization of blood vessels ultimately can lead to tissue infarction (death) [28]. Features such as hyper-dense middle cerebral artery (MCA), 
cerebral swelling caused by sulcal or ventricular effacement, and focal parenchymal hypoattenuation are most relevant to stroke assessment [29].

Ischemic core volume on baseline non-contrast CT (NCCT), CT perfusion (CTP), or diffusion-weighted magnetic resonance imaging (DWI) (Figure 6 shows the comparison of these modalities) is now widely used to drive key therapeutic decisions both in the early and late (beyond $6 \mathrm{~h}$ after last known time well) time windows [30]. Besides accessibility, speed, and patient tolerance, NCCT, when viewed in appropriate window width and window length, can detect early hyper-acute ischemic alterations which helps to predict both final outcome and the risk of secondary hemorrhagic changes [31]. DWI has been shown to contribute significantly to the early detection of acute ischemic infarction, which can be seen as a hyper-intense signal, due to the drop in diffusivity [32].

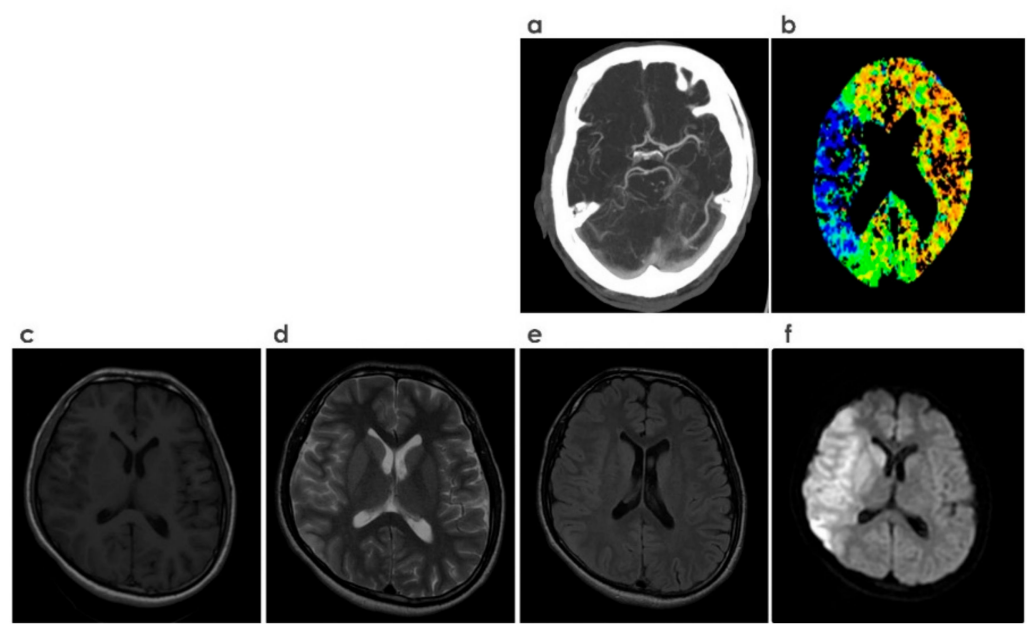

Figure 6. Various neuroimaging modalities. (a) CT Angiography, (b) CT Perfusion, (c) T1-weighted imaging, (d) T2-weighted imaging, (e) FLAIR (fluid attenuated inversion recovery), (f) DWI (diffusion weighted imaging). 
Table 3. Modalities at a Glance.

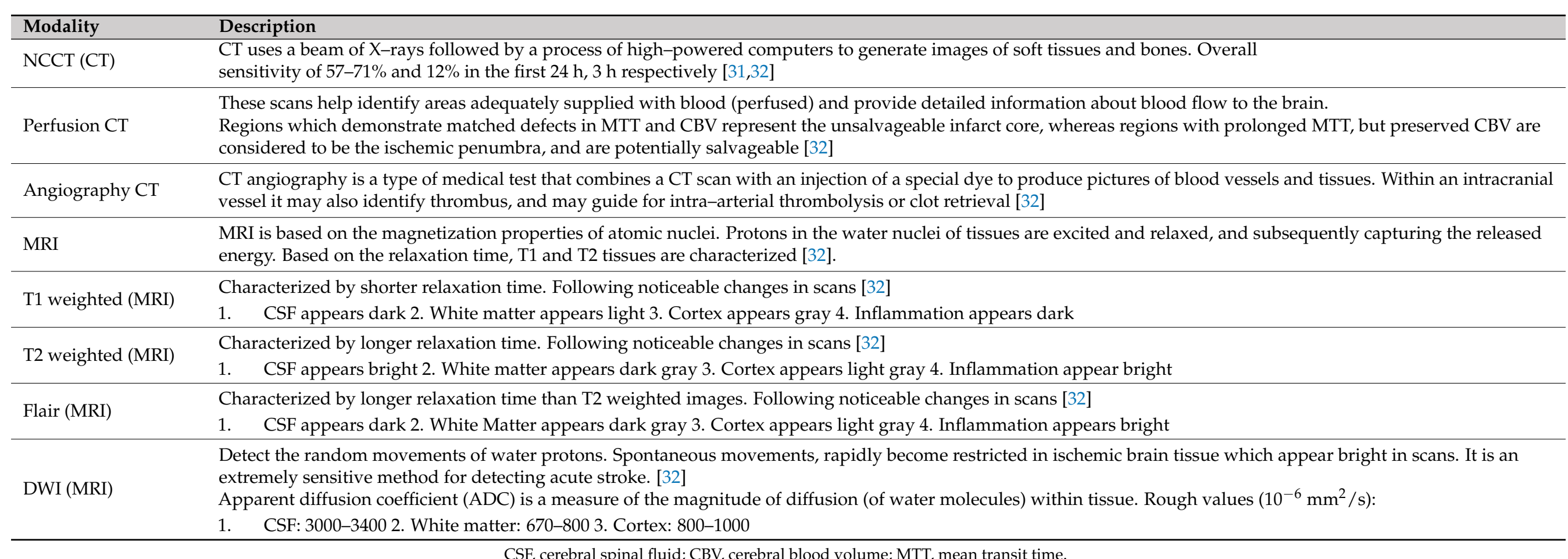




\subsection{Hemorrhagic Stroke}

Spontaneous extravasation of blood due to rupture of a vessel causes hemorrhagic stroke. The CT appearance of hemorrhage is proportional to the density of hemoglobin protein (relative to plasma concentrations) within the hematoma. Immediately following a vessel rupture, attenuation of $\mathrm{CT}$ is given in terms of Hounsfield units, which is a linear, quantitative measurement of radio density [33]. Contrast-enhanced CT angiogram (CTA) can identify patients at high risk of hemorrhage enlargement (HE) by revealing a 'spot sign', which indicates an active bleeding point within the hematoma. MRI, on the other hand, can detect previously resolved bleed and clinically silent cerebral microbleeds that are not detectable on CT. This is due to the detection of hemosiderin which is an end result of blood clot resolution [34]. Detection of hemosiderin provides information on previous history of hemorrhages that may have gone undetected [35]. Table 4 presents the radiological features shown by the considered modalities in different classes of stroke.

Table 4. Radiological features based on modalities.

\begin{tabular}{|c|c|c|c|c|c|c|}
\hline \multirow{2}{*}{$\begin{array}{c}\text { Stroke } \\
\text { Modality }\end{array}$} & \multicolumn{3}{|c|}{ Ischemic } & \multicolumn{3}{|c|}{ Hemorrhagic } \\
\hline & Acute (0-7 days) & $\begin{array}{c}\text { Subacute } \\
\text { (1-3 Weeks) }\end{array}$ & $\begin{array}{c}\text { Chronic } \\
\text { (>3 Weeks) }\end{array}$ & $\begin{array}{c}\text { Acute } \\
\text { (0-7 Days) }\end{array}$ & $\begin{array}{c}\text { Subacute } \\
\text { (1-3 Weeks) }\end{array}$ & $\begin{array}{c}\text { Chronic } \\
\text { (>3 Weeks) }\end{array}$ \\
\hline NCCT & $\begin{array}{l}\text { Loss of grey-white } \\
\text { matter } \\
\text { differentiation, and } \\
\text { hypo attenuation } \\
\text { (low density, } \\
\text { obstruction) of } \\
\text { deep nuclei }[31,32]\end{array}$ & $\begin{array}{l}\text { Attenuation of the } \\
\text { cortex }[31,32]\end{array}$ & $\begin{array}{l}\text { Hypo density } \\
\text { region }[31,32]\end{array}$ & $\begin{array}{c}\text { Hyper dense } \\
\text { with fluid levels } \\
\text { [33] }\end{array}$ & $\begin{array}{l}\text { Less intense } \\
\text { with } \\
\text { ring-like } \\
\text { profile [33] }\end{array}$ & $\begin{array}{l}\text { Iso dense or } \\
\text { modest } \\
\text { confined } \\
\text { hypo } \\
\text { density [33] }\end{array}$ \\
\hline T1 & Low T1 signal [32] & Low T1 signal [32] & $\begin{array}{c}\text { Low T1 signal } \\
\text { [32] }\end{array}$ & $\begin{array}{c}\text { Iso intensity or } \\
\text { slight hypo } \\
\text { intensity with } \\
\text { thin hyper } \\
\text { intense rim in } \\
\text { the periphery } \\
{[32,33]}\end{array}$ & $\begin{array}{c}\text { Hyper } \\
\text { intensity } \\
{[32,33]}\end{array}$ & $\begin{array}{c}\text { Нyро } \\
\text { intensity } \\
{[32,33]}\end{array}$ \\
\hline $\mathrm{T} 2$ & $\begin{array}{l}\text { infarct remains } \\
\text { Hyper intense [32] }\end{array}$ & $\begin{array}{c}\text { Hyper intensity } \\
{[32]}\end{array}$ & $\begin{array}{c}\text { High T2 signal } \\
\text { [32] }\end{array}$ & $\begin{array}{c}\text { Hypo intense } \\
\text { with hyper } \\
\text { intense } \\
\text { perilesional rim } \\
{[32,33]}\end{array}$ & $\begin{array}{c}\text { Hyper } \\
\text { intensity } \\
{[32,33]}\end{array}$ & $\begin{array}{c}\text { Нypo } \\
\text { intensity } \\
\text { [32] }\end{array}$ \\
\hline DWI & $\begin{array}{l}\text { Decreased ADC } \\
\text { values with } \\
\text { maximal signal } \\
\text { reduction within } 1 \\
\text { to } 4 \text { days marked } \\
\text { with hyper } \\
\text { intensity [32] }\end{array}$ & $\begin{array}{l}\text { First ADC values } \\
\text { rise and return } \\
\text { close to baseline, } \\
\text { despite normal } \\
\text { ADC values } \\
\text { irreversible tissue } \\
\text { necrosis is present } \\
\text { (DWI remains } \\
\text { hyper intense) [32] }\end{array}$ & $\begin{array}{c}\text { ADC signal } \\
\text { high [32] }\end{array}$ & ADC: 0.70 [35] & $\begin{array}{c}\text { ADC: } 0.72 \\
{[35]}\end{array}$ & $\begin{array}{c}\text { ADC: } 2.56 \\
{[35]}\end{array}$ \\
\hline
\end{tabular}

ADC, apparent diffusion coefficient.

\section{Machine Intelligence in Lesion Segmentation and Stroke Detection}

Lesion segmentation and identification of brain abnormality has long been a subject of research, and many resulting developments have been made. Computer aided techniques with statistical analysis have improved the process and model accuracy. However, as many of these tools rely on human intervention or for crafting specialized features, these methods are computationally expensive and suffer from a lack of generalizability. In contrast, machine learning algorithms can learn from hidden data and offer great flexibility. However, these too have the problem of addressing handcrafted features and being specific to the 
available dataset. Hence, it is prudent to develop a technique with many parameters to learn and acquire the important features, thereby sparing manual work. Introduction of such systems in medical practice, if accurate and sensitive, may be cost effective, freeing clinicians to focus on other areas of patient management. In regions where skilled radiologists are limited, an automated technique can improve accessibility and equity in high quality medical care. Most importantly, they have the ability to improve the early detection of stroke and facilitate improved outcomes guided by accurate neuroimaging [36].

These deep learning networks are known as global function approximators, making them ideal tools for the case with non-uniform relationships between parameters. As these have learnable parameters in multitude, they are highly efficient in capturing minute and salient information [37]. However, there are some difficulties, the first being that they are 'data hungry', and sufficient data may, in some cases, be difficult to provide, leading to the need for data augmentation. Secondly most networks are massive in terms of layers and hence the changes in derivatives might not effectively initiate earlier neurons. Thirdly, as the computations depend on decision logic, higher-level computational machinery is required, such as the use of graphics processing units (GPUs) or tensor processing units (TPUs). Due to rapid growth in healthcare and computational infrastructure, deep learning in many ways has stood the test of time to emerge as an efficient tool for such applications.

The following sections provide an overview on the current techniques with CT and MRI as primary modalities for ischemic and hemorrhage stroke detection.

\subsection{Computer Aided-Statistical Techniques}

Schemes with detection technique aided by computer processing (CAD) can help identify patterns or abnormality that might be missed in preliminary clinical diagnosis, and with the automatic feature extraction may improve disease detection. These can be broadly grouped into two types: (a) region of interest (ROI) detection followed by stroke prediction, and (b) segmentation. In the following section we discuss relevant papers in the same order.

\subsubsection{CT Based Methods}

Techniques from the past decade have evolved tremendously from region identification, feature extraction through image enhancement through computer to identify stroke, and early detection which is critical to guide most appropriate therapies and improve health care outcomes. Tang et al., proposed a way to isolate the region of interest of geometric shapes to analyze CT scans for prompt discovery of ischemic stroke [38]. The algorithm contains a series of filters using radii of pixel to obtain the region of interest and produce a binary mask. The technique performs the identification for detecting brain midline using statistical analysis. Sajjadi et al., proposed a filter bank algorithm (adaptive partial median filer), called the àtrous algorithm to clear the noise and enhance the image for detecting early signs of ischemic stroke [39].

To deal with case of misses by experts, due to the low sensitivity of NCCT in detecting cerebral infarctions, Nowinski et al., proposed a quick, less intensive and automatic method to detect, isolate and assess ischemic infarct from a single NCCT scan [40]. Filho et al., proposed a method based on extracting radiological density patterns of the brain to detect and categorize the occurrence of stroke. Five classifiers were applied and compared for ischemic stroke detection in CT images [41]. Flottman et al., experimented with thresholdfree prediction of brain infarct from CTP imaging in case of core to penumbra lesion mismatch [42]. Sakai et al., compared Bayesian versus singular value deconvolution for estimation of ischemic core volume as a discriminant. He used a CTP-CBF threshold $<30 \%$ of a normal brain [43]. As early identification of stroke can be tremendously advantageous, Lo et al., developed a feature set extracted and enhanced by the Ranklet transform to feed the ML classifier for the early detection of hyper-acute ischemic stroke [44]. Shervin K et al., presented a study to determine the finest CTP parameters and associated threshold to clearly discriminate between benign and at-risk penumbra region without reperfusion [45]. 
Kheradmand et al., conducted a study which showed that in cases of operfusion CT, Time to peak when compared with mean transit time is a more accurate parameter to identify the "at-risk" tissue [46]. Kawiorski M et al., conducted a study which emphasizes the utility of perfusion CT to identify the potentially salvageable tissues by corroborating the presence of correlation between the clinical and radiological aspects [47].

Bhaduria et al., presented a unique region growing based segmentation technique wherein fuzzy c mean facilitates identification of active contour and thereafter propagation through region-based method for intracranial hemorrhage (ICH) detection [48]. Haan et al., proposed the clusterize algorithm as a semi-automated lesion segmentation approach to speed up the demarcation process without reducing precision [49]. Yahiaoui et al., enhanced brain CT imagery using the Laplacian pyramid (LP) and then a Fuzzy C mean clustering algorithm for segmentation of ischemic stroke [50]. In grayscale thresholdbased techniques, Reboucas et al., proposed a new and more stable level set approach for stroke segmentation in CT brain imagery [51]. Kumar et al., proposed an entropy based unsupervised segmentation techniques for brain ICH [52]. Vasconcelos et al., proposed a faster method for extracting featuring using adaptive brain tissue density analysis coupled with federated learning to aid in stroke detection and classification [53].

\subsubsection{MRI Based Methods}

A standard MRI for acute stroke protocol consists of multiple sequences, from basic T1-weighted, T2-weighted, fluid attenuated inversion recovery (FLAIR), diffusion weighted imaging (DWI), susceptibility-weighted imaging (SWI) and MR angiography (MRA) (doi:10.1148/rg.325115760). This multispectral application is time consuming. In this regard, Nabizadeh et al., proposed an intensity-based segmentation technique optimized by gravitational algorithm for automatic stroke detection and segmentation using single-spectral MRI [54]. Ghosh et al., performed comparative analysis of three techniques for segmentation, namely modified watershed segmentation (MWS), symmetry integrated region growing (SIRG), and hierarchical region splitting (HRS) for the detection of hypoxic ischemic injuries [55]. In the case of segmentation, a lack of a sharp boundary delineation hinders and delays the identification process. Cauley et al., tested a hypothesis and proved that image intensity inhomogeneity provides a sign for identifying the subtle hypo-density regionals which, in turn, is characteristic of ischemic infarct [56]. Ledig C et al., proposed a probabilistic framework for automatic segmentation of MRI using "multi-atlas label propagation" [57]. Farsani et al., proposed a fully automated lesion segmentation method, which works on diffusion restriction characteristics of the acute stroke images [58]. Moeskops et al., proposed a voxel based automatic segmentation into several tissue classes using CNN with different patch sizes and kernel sizes to acquire multi-scale information about each voxel [59]. Oula et al., presented a simultaneous processing approach which combines brain segmenting techniques with a novel spatial lesion model for identifying distinct brain structures using a restricted Boltzmann machine [60]. Si et al., proposed a wavelet transform based supervised segmentation technique optimized by Grammatical Bee Colony algorithm [61]. Tom et al., presented a probabilistic technique for calculating intensities of both normal and at risk (pathological) tissue without the need of a training set [62]. Ji et al., performed accurate segmentation of brain tissue from the MR image based Gaussian mixture model (GMM) [63]. Kamnitsas et al., proposed an architecture which addresses the challenging task of brain lesion segmentation, making it more efficient and adaptive to the class imbalance problem [64]. Figure 7 presents a generalized pictorial representation of the pipeline of processes, and a summary of all techniques is presented in Table 5. 


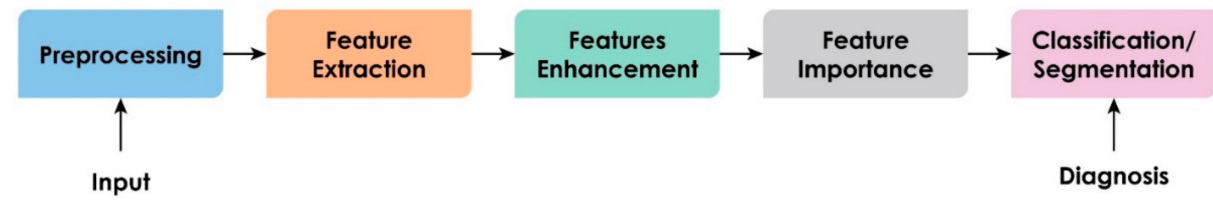

Figure 7. General block diagram of a typical ML-based CAD system.

Table 5. Summary of computer aided statistical techniques for lesion segmentation and stroke detection.

\begin{tabular}{|c|c|c|c|c|}
\hline Articles & Modality & Technique & Outcome & Year \\
\hline Tang et al. [38] & $\mathrm{CT}$ & $\begin{array}{l}\text { Image texture analysis through Circular } \\
\text { Adaptive Region of Interest method }\end{array}$ & $S_{\text {ROC }}:[0.99-0.94]$ & 2011 \\
\hline Sajjadi et al. [39] & $\mathrm{CT}$ & $\begin{array}{c}\text { Translation-invariant wavelet for image } \\
\text { enhancement }\end{array}$ & Higher information image extracted & 2011 \\
\hline Nowinski et al. [40] & NCCT & $\begin{array}{l}\text { Analyzing hemisphere attenuation values } \\
\text { using percentile difference ratios }\end{array}$ & $\begin{array}{c}\mathrm{S}_{\text {Acc }} \text { is } 83.2 \% \text {. The early detection } \\
\text { accuracy }(<3 \mathrm{~h}) \text { is } 78.4 \% \text {. }\end{array}$ & 2013 \\
\hline Filho et al. [41] & $\mathrm{CT}$ & Analysis of brain tissue density & & 2017 \\
\hline Flottman et al. [42] & $\mathrm{CT}$ & Novel threshold free method & & 2017 \\
\hline Lo et al. [44] & NCCT & $\begin{array}{l}\text { Local contract enhancement using Ranklet } \\
\text { Transformation and probability based } \\
\text { detection }\end{array}$ & $\begin{array}{l}\text { GLCM Ranklet } \\
\mathbf{S}_{\text {ACC }} 71 \% 81 \%\end{array}$ & 2019 \\
\hline Bhaduria et al. [48] & CT & $\begin{array}{l}\text { Segmenting through the features of both } \\
\text { fuzzy clustering and region-based active } \\
\text { contour model }\end{array}$ & $S_{\mathrm{DC}}: 0.92$ & 2014 \\
\hline Haan et al. [49] & $\begin{array}{l}\text { CT, DWI, } \\
\text { T2FLAIR }\end{array}$ & $\begin{array}{c}\text { Clustering algorithm for lesion } \\
\text { demarcation in AIS }\end{array}$ & $\begin{array}{l}\text { Reduced processing time to on } \\
\text { average } 17.8 \mathrm{~min} / \text { patient }\end{array}$ & 2015 \\
\hline $\begin{array}{l}\text { YAHIAOUI et al. } \\
\text { [50] }\end{array}$ & $\mathrm{CT}$ & $\begin{array}{c}\text { Differentiation of brain pathology area } \\
\text { (hypodense) from its adjacent normal } \\
\text { parenchym (i.e., contrast enhancement) } \\
\text { using Laplacian Pyramid }\end{array}$ & $\begin{array}{l}\text { Laplacian Pyramid algorithm gives } \\
\text { Better and faster }(10.46 \mathrm{~s}) \text { result than } \\
\text { DWT, especially in small sized lesions. }\end{array}$ & 2016 \\
\hline Reboucas et al. [51] & $\mathrm{CT}$ & $\begin{array}{l}\text { Level set based approach on brain } \\
\text { densities (radiological) method to } \\
\text { generate stroke segmentation }\end{array}$ & $\begin{array}{c}\text { Segmentation time and } \mathrm{S}_{\mathrm{ACC}} \\
\text { LSBRD (proposed) } 1.76,99 \% \\
\text { Watershed } 3.10,92 \% \\
\text { Region Growing } 4.81,93 \%\end{array}$ & 2017 \\
\hline Kumar et al. [52] & $\mathrm{CT}$ & Entropy based segmentation & $\mathbf{S}_{\mathrm{ACC}}: 99.87$ (avg) & 2020 \\
\hline $\begin{array}{l}\text { Vasconcelos et al. } \\
\text { [53] }\end{array}$ & $\mathrm{CT}$ & Adaptive Brain Tissue Density Analysis & $C_{\text {ACC }}: 98.13 \%$ & 2020 \\
\hline $\begin{array}{l}\text { Nabizadeh et al. } \\
\text { [54] }\end{array}$ & MRI & $\begin{array}{l}\text { Histogram-based gravitational } \\
\text { optimization algorithm }\end{array}$ & $S_{\text {ACC }}: 91.5 \%($ strokes) & 2014 \\
\hline Ghosh et al. [55] & & $\begin{array}{l}\text { Hierarchical Region Splitting, Symmetry } \\
\text { Integrated Region Growing and Modified } \\
\text { Watershed Segmentation }\end{array}$ & & 2014 \\
\hline Ledig et al. [57] & MRI & $\begin{array}{l}\text { Refinement using Multi-Atlas Label } \\
\text { based context with } \\
\text { Expectation-Maximization. }\end{array}$ & $64.7 \% \mathbf{S}_{\text {ACC }}$ using acute-phase & 2015 \\
\hline Farsani et al. [58] & MRI & Diffusion restricted characterisitics & $\mathrm{C}_{\mathrm{ACC}}: 73 \%$ & 2016 \\
\hline Moeskops et al. [59] & MRI & $\mathrm{CNN}$ & $\mathrm{S}_{\mathrm{DC}}: 0.84-0.91$ & 2017 \\
\hline Ji et al. [63] & MRI & Gaussian Mixture Model & $\mathbf{S}_{\mathrm{ACC}}: 5 \%$ more than baseline model & 2017 \\
\hline $\begin{array}{c}\text { Kamnitsas et al. } \\
\text { [64] }\end{array}$ & MRI & $\begin{array}{l}\text { A } 11 \text { layered dual pathway architecture } \\
\text { for joint processing of adjacent image } \\
\text { patches (DeepMedic) }\end{array}$ & $\begin{array}{c}\mathrm{S}_{\mathrm{DC}} \text { on training data of BRATS } 2015 \\
\text { DeepMedic + CRF } 89.8 \\
\text { DeepMedic } 89.7\end{array}$ & 2017 \\
\hline
\end{tabular}

$\mathrm{S}_{\mathrm{ROC}}$, receiver output receiver; GLCM, gray-level co-occurrence matrix; $\mathbf{S}_{\mathrm{ACC}}$, segmentation accuracy; $\mathbf{S}_{\mathrm{DC}}$, segmentation dice coefficient, $\mathrm{C}_{\mathrm{ACC}}$, classification accuracy. 


\subsection{Machine Learning Methods}

3.2.1. Ischemic Stroke

\section{CT based methods:}

For CT images, an ischemic stroke appears as a dark or low attenuation (hypodense) region, well contrasted against its surroundings. For early detection, manual processing via a clinical expert has traditionally been the most effective, but it is time-consuming (especially under the time pressures of acute stroke management). Hence, an emphasis is given to automation of detection using machine learning techniques. Rajini et al., developed an approach for segmentation with amalgamation of texture analysis and the midline shift tracing algorithm [65]. Quantifying cerebrospinal fluid (CSF) volumetric changes over time is a potential biomarker for cerebral edema, and these studies performed this by using ML [66,67]. Guberina et al., performed the Alberta stroke program using ML techniques to detect early infarction sign [68]. As the features extracted from MRI yield better results, we see a major use of ML techniques with MRI.

\section{MRI based methods:}

To date, MRI is the most sensitive modality to detect hyperacute stroke by determining early cellular swelling due to ischemia. Brain ischemia produces effects that are time variant. Hence, dynamic changes are seen in MRI, from early hyperacute (0-6 h of onset) to chronic ( $\geq 3$ months) staging $[69,70]$. Teruyuki et al., found that in case of acute stroke, mismatch of abnormalities between images of perfusion-weighted MR and DWI could help identify the penumbral region [71]. Maier et al., presented a comparison study of different ML based classification methods for ischemic stroke lesion segmentation [72]. Mitra et al., explored the probabilistic method of Bayesian-Markov random field (MRF) for segment (FLAIR) MRI and employed random forests (RFs) to extract highly probable lesion areas [73]. Bharathi et al., explored ways to enhance segmentation quality using handcrafted and unsupervised techniques and derived features [74]. Yoo et al., performed a study to determine optimum thresholds of MRI modality parameters to aid the decision to provide reperfusion therapy on the onset of stroke symptoms [75]. Maier et al., proposed an automatic method of extra tree forests for voxel-based classification with an emphasis on reproducibility and robustness to noise [76].

Ensemble techniques have been widely employed for better results. Mark et al., applied five ML algorithms (viz. generalized linear, additive model, adaptive boosting, SVM, and RFs) to outline intense cerebral ischemic tissues that can recover after reperfusion [77]. Bagging technique such as RFs have been popular amongst most of the detection work, perhaps due to their high resilience to variance. Muschelli et al., and Qaiser et al., experimented with RF customized features (local moment details, MRI's scan, smooth and median intensities) to predict the presence of ischemic penumbra and segmentation [78,79]. Fusing and cascading classifiers distributed across reference space and grouped to be classified with high-level region-specific RFs have yielded good results [80]. Hanna et al., presented a technique for segmentation using RFs with context-based clustering techniques [81]. Jerman et al., integrated an unsupervised segmentation technique with RFs (supervised) [82]. Mckinley et al., proposed an automatic method for segmenting ischemic penumbra using spatial and textural features on "Segmentation Forests" [83]. Robben et al., proposed a segmentation technique using cascading extremely randomized forest classifiers [84]. Chen $\mathrm{H}$ et al., proposed a segmentation technique using dense conditional random fields to enhance the probability maps which are then used to train RFs [85]. To deal with the issue of model generalization and the inability to be specific to cater to the highly dynamic expressions of pathology, Goetz et al., proposed a methodology which adaptively samples optimal images from a training set to train classifiers (thereby supporting heterogeneous databases) [86]. Few have experimented with other supervised techniques either distance based ( $k$-nearest neighbor) or probabilistic (Gaussian Naïve Bayes) [87,88]. Karthik et al., utilized the discrete curvelet transformation with a few statistical parameters as features on different scales to train the RBF kernel SVM model and 
ANN [89]. ML models have the tendency to become complex in lieu of better performance. Pereira et al., proposed an unsupervised technique (RBM) for feature learning and to feed the RF classifier for penumbra estimation and evaluation of tumor segmentation [90]. Lin et al., conducted a study to assess quality and identify potentially erroneous measurements due to the presence of outliers. He evaluated and confirmed the suitability of a densitybased detection method [91]. Subudhia et al., have used Delaunay triangulation (DT) for optimizing segmentation and tuned the parameters through "Fractional Order Darwinian particle swarm optimization" (FODPSO), for automatic segmentation of stroke lesions [92]. Table 6 contains a summary of these techniques.

Table 6. Summary of various ML techniques applied for stroke detection (ischemic) and segmentation.

\begin{tabular}{|c|c|c|c|c|}
\hline Articles & Modality & Technique & Outcome & Year \\
\hline Filho et al. [41] & CT & $\begin{array}{l}\text { Feature extraction based on density patterns (radiological) and } \\
\text { classification of strokes through Bayesian, SVM, kNN, MLP, and } \\
\text { OPF classifiers }\end{array}$ & $\begin{array}{l}\text { Fastest extraction time } \\
\text { I }_{\text {ACC }}: 99.30 \%\end{array}$ & 2017 \\
\hline Rajini et al. [65] & CT & $\begin{array}{l}\text { Symmetry (mid line shift) based segmentation; image texture } \\
\text { analysis using GLCC and classification using SVM, k-NN, } \\
\text { ANN, decision tree }\end{array}$ & SVM I ACC $: 98 \%$ & 2013 \\
\hline Maier et al. [72] & MRI & $\begin{array}{l}\text { Generalized Linear Models, RFs and CNN are evaluated and } \\
\text { compared with each other for sub-acute ischemic stroke patients }\end{array}$ & AdaBoost $\mathbf{I}_{\mathbf{D C}}: 0.69$ & 2015 \\
\hline Mitra et al. [73] & FLAIR MRI & Bayesian-Markov Random Field and RF & I DC: $_{0.60 \pm 0.12}$ & 2014 \\
\hline Bharathi et al. [74] & $\begin{array}{l}\text { MRI T1, T2, } \\
\text { DWI and } \\
\text { FLAIR }\end{array}$ & $\begin{array}{l}\text { Feature Extraction using GLCM and unsupervised extraction } \\
\text { Kmeans clustering; and training RF classifier for detection of } \\
\text { ischemic stroke lesion }\end{array}$ & $\mathbf{I}_{\mathbf{D C}:} 0.88 \mathbf{I}_{\mathrm{ACC}} 0.82$ & 2019 \\
\hline Maier et al. [76] & $\begin{array}{l}\text { T1w, T2w, } \\
\text { FLAIR and } \\
\text { DWI }\end{array}$ & Extra Tree Forest framework for voxel-wise classification & $\mathbf{I}_{\mathbf{D C}}: 0.65 \pm 0.18$ & 2015 \\
\hline $\begin{array}{l}\text { McKinley et al. } \\
\text { [83] }\end{array}$ & MRI T1, T2 & $\begin{array}{l}\text { Spatial } \\
\text { Random Forest }\end{array}$ & $\begin{array}{l}\text { ISLES (leave one out) } \\
\text { I }\end{array}$ & 2015 \\
\hline Robben et al. [84] & $\begin{array}{l}\text { T1w- and } \\
\text { T2w, Flair and } \\
\text { DWI }\end{array}$ & cascaded extremely randomized trees & $\begin{array}{c}\mathbf{I}_{\mathbf{D C}} \\
\text { SISS } 0.57 \pm 0.28 \\
\text { SPES } 0.82 \pm 0.07\end{array}$ & 2016 \\
\hline Chen et al. [85] & MRI & random forests (cascaded) with dense conditional randomfields & $\begin{array}{c}\text { ISLES 2015/BRATS } 2018 \\
\mathbf{I}_{\text {DC }} \text { of } 0.51 \pm 0.29 / 0.86\end{array}$ & 2020 \\
\hline Griffanti et al. [87] & T2 and FLAIR & k-nearest neighbor & ICC: 0.99 & 2016 \\
\hline Griffis et al. [88] & $\mathrm{T} 1$ & Gaussian naïve Bayes & $\mathbf{I}_{\mathbf{D C}} 0.66$ & 2016 \\
\hline Karthik et al. [89] & MRI & $\begin{array}{l}\text { Multidirectional features based on Discrete curvelet transform } \\
\text { and watershed algorithm for fetching the ROI and then } \\
\text { applying support vector machines to develop the classification } \\
\text { system. }\end{array}$ & $\mathbf{I}_{\mathrm{ACC}} 99.1 \%$ & 2017 \\
\hline Pereira et al. [90] & MRI & Unsupervised feature learning through RBM with RF classifier & $\mathbf{I}_{\mathbf{D C}} 0.81 \pm 0.84$ & 2018 \\
\hline Lin et al. [91] & CT & $\begin{array}{l}\text { DBSCAN, hierarchical DBSCAN (HDBSCAN) } \\
\text { and local outlier factor (LOF) for identification of erroneous } \\
\text { stroke detection }\end{array}$ & DBSCAN (Avg) I ICC 96.9 & 2019 \\
\hline $\begin{array}{l}\text { Subudhia et al. } \\
\text { [92] }\end{array}$ & MRI & $\begin{array}{c}\text { Delaunay triangulation based segmentation optimized by } \\
\text { Darwinian particle swarm optimization }\end{array}$ & $\mathbf{I}_{\mathrm{ACC}}$ of 0.95 & 2018 \\
\hline Peixoto et al. [93] & $\mathrm{CT}$ & SCM, SVM, MLP & $\mathbf{I}_{\text {SPEC }}=99.1 \%[$ highest $]$ & 2018 \\
\hline Garg et al. [94] & $\begin{array}{c}\text { Electronic } \\
\text { Data (NLP) }\end{array}$ & $\begin{array}{l}\text { Classification of Ischemic Stroke Subtype (TOAST) using ML } \\
\text { (RF, GBM, KNN, XGBOOST, SVM, Extra Trees) and NLP }\end{array}$ & $\begin{array}{l}\text { Kappa stacking: } \\
\text { combined data }=0.57\end{array}$ & 2019 \\
\hline
\end{tabular}

GLCM, gray-level co-occurrence matrix; DC, dice coefficient; $\mathbf{I}_{\mathbf{D C}}$, ischemic stroke dice coefficient; $\mathbf{I}_{\mathrm{ACC}}$, accuracy; $\mathbf{I}_{\mathbf{I C C}}$, intra class correlation coefficient; $\mathbf{I}_{\text {PREC }}$, ischemic precision; $\mathbf{I}_{\mathbf{F S}}$, ischemic FScore; $\mathbf{I}_{\text {SPEC }}$, ischemic specificity; $\mathbf{I}_{\mathbf{S E N S}}$, ischemic sensitivity.

\subsubsection{Hemorrhagic Strokes}

Intracranial hemorrhage is defined as bleeding that occurs inside the brain parenchyma. Chen et al., showed an interesting way for detection of brain hemorrhagic diagnosis using Internet of Things [95]. Gillebert et al., present a method to automatically delineate infarct and hemorrhage in stroke CT imagery [96]. The process involves normalized CT images 
from stroke patients into a template space, and the subsequent voxel-wise comparison with a group of control CT images for defining areas with hypo- or hyper-intense signals. Diagnosing ICH is straight forward. However, identifying early hemorrhagic transformation in ischemic stroke can be challenging. Thrombolysis (fibrinolytic therapy), is the process of breakdown of clots (blood) formed in vessels using medication. This could be lifesaving in case of ischemic stroke but disastrous in cases of hemorrhage. Hence, there must be a method to first identify the stroke before administering thrombolysis. Bentley et al., conducted a study with the ML (SVM) model for predicting the presence of ICH [97]. In Figure 8, the stages for lesion segmentation, identification, and classification of stroke regions for machine learning techniques are shown. Table 6 contains a summary of these techniques.

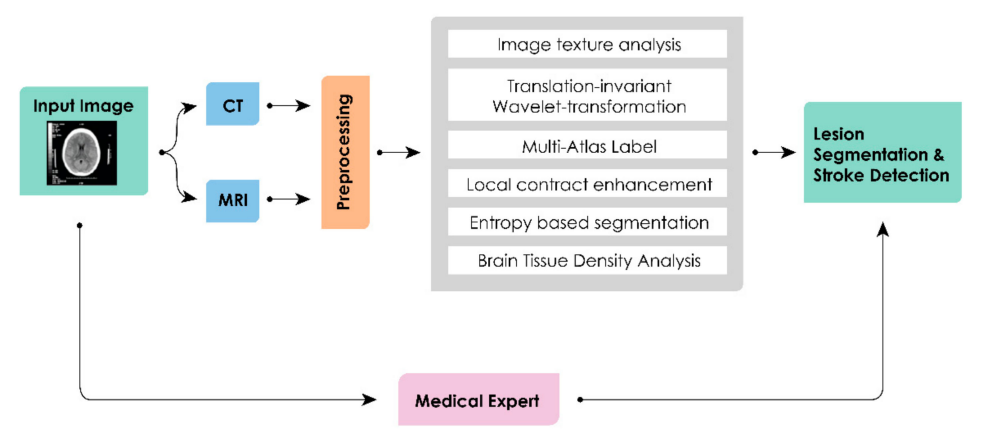

Figure 8. Generalized stages for lesion segmentation, identification, and classification of stroke regions.

\subsection{Deep Learning Methods}

\subsubsection{Ischemic Strokes}

\section{CT based methods:}

CNNs are widely useful for adaptability and recent experiments provide evidence of good results using 3D CNN, which captures volumetric information. Chin et al., addressed the difficult task of segmenting acute ischemic lesions, due to their subtle nature as compared with traditional CNN [98]. Identification of highly dynamic texture and intensity variations in pathology is a difficult task using NCCT given the poor visibility. Lisowska et al., investigated the betterment in working accuracy of CNN when appended with spatial information (ATLAS). Although this network performed better due to this incorporation, it was found to be less useful in the case of ischemia [99]. Abulnaga et al., extracted contextual information using a pyramid pooling net (pyramid scene parsing network) [100]. Lucas et al., developed a 3D U-net to predict the final form of lesion with trained clinical knowledge (core and penumbra shapes) represented in lower dimension by a convolutional auto-encoder [101].

Many variants of recurrent networks have been utilized for stroke detection. Vargas et al., built a Res-CNN stacked with a (long short-term memory (LSTM) layer) to check the presence of ischemic stroke [102]. Barman et al., devised a deep symmetry sensitive network (in lines of Siamese networks and inception modules) to analyze symmetrical information [103]. Clèrigues et al., used asymmetric res-encoder-decoder model CT imagery for detecting core infarcts using 2D patches [104]. Shinohara et al., proposed a DCNN model to identify a hyperdense middle cerebral artery (a clinical sign indicating blockage of the artery) to segment regions of ischemic lesion [105]. Due to the wide scale of hypo densities in CT images, it is prudent to utilize an ensemble technique for better generalization and specific results. Barros et al., used three different CNNs for segmentation of subtle, intermediate, and clear hypo-dense lesions. It was seen to be reliable and provided excellent correlation with the reference infarct volume [106].

Oman et al., explored the possibility of appending cerebral hemispheric comparison CTA and NCCT as input in addition to CTA to possibly improve the performance of CNN in the detection of AIS. It was reported to have two-fold benefits first being the increased 
specificity in ischemic lesion detection specificity and second to decrease the number of false positives [107]. Hu et al., proposed a faster, efficient network for lesion segmentation [108]. Islam et al., proposed a training segmentation model using adversarial learning, as this would detect and rectify higher order inconsistencies between the segmentation maps produced by ground-truth and the segmentor. The model consists of a segmentor (generative model) which generates the synthesized model, and a discriminative model that estimates the likelihood of a sample being from ground truth data [109]. Bertels et al., proposed a CNN model with the data present in a nearby (contra-lateral side) voxel for voxel-wise lesion segmentation of the core lesion [110]. Kuang et al., proposed a novel multi-task learning approach i.e., EIS-Net, to segment early Infarct and score "Alberta Stroke Program Early CT Score (ASPECTS)" simultaneously on baseline NCCT scans of AIS patients [111]. Avetisian et al., experimented with altered U-Net CNN architecture by slimming the encoder for the detection of stroke [112]. Robben et al., used a data driven and deconvolution free approach to have a deep learning network to predict the final infract volume [113]. Wang et al., proposed a method to extract features using the RF classifier for automatic stroke lesion 3D segmentation [114].

\section{MRI based methods:}

MRI, with its inherent excellent soft tissue contrast resolution of the whole brain, offers a simple post-processing operation and provide the flexible ability to simultaneously perform diffusion imaging. However, co-existing MRI findings such as underlying cerebral deep white matter chronic micro-ischemia and indistinct stroke area can sometimes be difficult to segment. CNN have proved to better performing for semantic segmentation [115]. Havaei et al., proposed a CNN based two-pathway framework trained directly on modality, where each path focused on smaller and larger details [116]. Stier et al., built and evaluated a DL model to predict tissue survival outcome based on sampled (randomly) local patches of the hypo-perfusion (Tmax) feature measured immediately after the onset of symptoms [117]. Dou et al., proposed an automatic 3D CNN model for performing a detection operation using a cascading framework [118]. Choi et al., proposed an ensemble of DNNs for the technical tasks of prognosis of post-treatment in case of stroke. This study gave a multiphase learning technique to address the class imbalance problem [119]. As this process is deep and heavily parametrized, and would certainly take a longer time to converge, there are scopes to enhance the computational efficiency and improve time/space constraints.

Although diffusion-weighted MR imaging (DWI) is sensitive to the lesions, manually localizing and quantifying them is costly and challenging in terms of time and resources. Wang et al., proposed an attention-based DNN with synthesized pseudo-DWI from perfusion maps to obtain superior image quality for better segmentation [120]. Chen et al., proposed model framework consists of two CNNs for segmentation (automatic) of DWI based stroke lesions in DWI. The architecture contains an ensemble of two DeconvNets for detection of lesion, followed by a second CNN (MUSCLE Net) for refinement and identifying and removing false positives [121]. Lucas et al., studied the use of classical fully-connected neural networks (FC-NN) (151 features) based on handcrafted featuring, and compared the results with DCNN and RF models in terms of accuracy and convergence time. FCNN achieved much shorter runtimes [122]. Alex et al., proposed a de-noising autoencoder model for unsupervised feature learning of brain lesion detection, segmentation, and reducing false positives [123]. Giacalone et al., employed the local spatial information (temporal) for prediction of final lesion [124]. Perfusion imaging is essential to assess penumbra area and infarcted core due to its ability to measure blood flow, transition times and dispersion. Lucas et al., implemented an extension of U-Net, and added skip connections after an alternative $3 \times 3$ Conv Block. Surface distance proved to be more useful than pixel/voxel matching for irregular shape and to avoid low scores [125]. Bento et al., performed a study and build an architecture for identification of atherosclerosis areas [126]. Song et al., proposed a novel generative technique consisting of extractor (features from CTP), generators (DWI based features) and segmentor [127]. Liu et al., 
proposed a 2D-slice-based segmentation method with a residual-structured FCN (ResFCN) on the multi-spectral MRI process. Many blocks of CNN were involved for better feature extraction [128]. Zhang et al., proposed a deep 3D CNN for automatic segmentation by extended DenseNets to 3D and tapped their potential on AIS segmentation from DWI. They employed a Deep supervision technique and Dice objective function to improve optimization [129].

Chen et al., proposed a novel voxel-wise residual network (VoxResNet) with a set of effective training schemes to address segmentation in the complicated anatomical environment of the brain and the large variations of brain tissue [130]. Li et al., presented a 2D ensemble FCNN based architecture to spot hyper-intense regions in fluid attenuated inverse recovery (FLAIR) and T2 weighted imagery [131]. It is seen to achieve best results on hand crafted features, which in turn are complex and often lack the ability to distinguish between affected and normal tissue. Praveen et al., proposed a stacked sparse auto encoder framework for automatically learning and selecting features followed by the SVM classifier to accurately segment stroke lesions from brain MR images [132]. Due to the limited number of labelled and high-resolution scans, currently many investigators generate synthetic data and train the model adversely. Alex et al., proposed a semi-supervised technique with a generative adversarial network (GAN) for brain lesion segmentation [133]. Li et al., proposed an 2D dilated deep residual network to capture contextual information for segmentation task [134]. Luna et al., proposed a novel 3D CNN based with transition layers between encoding and decoding process to increase the impact of features maps in latter phase [135]. Winzeck et al., investigated whether an ensemble of convolutional neural networks trained on a multi-parametric DWI (MRI) mapping outperforms single networks trained on solo DWI parametric maps [136]. Liu et al., proposed a DCCN (Res-CNN) to automatically segment acute ischemic stroke area from multi-modality MRIs. In contrast to the single modality version, use of multimodality helps to improve segmentation performance [137]. Karthik et al., proposed a supervised DFCN, with leaky ReLU as the activation in the last two layers of the network for a precise reconstruction (absent in U-Nets) [138]. Li et al., presented a unique end-to-end brain tumor segmentation method by modifying the up-skip connection between the encoder and decoder, and adopting the inception module $(7 \times 7$ high receptor convolutional layers) in each block to help the network learn richer representations [139]. Malla et al., explored the scope to evaluate the impact of enhanced ML techniques, advancements, transfer learning, and post-processing in the segmentation of stroke areas. [140].

These studies underscored the importance of multi-scale features and contextual features and ways to capture long range dependencies [141,142]. Liu et al., proposed a DCNN for stroke MRI based segmentation to address overfitting [143]. Chin et al., showed that ensemble techniques like cascading could be used for post stroke analysis to improve results [144]. Studies have been performed to perform segmentation using neighborhood or symmetry information $[145,146]$. Rajan et al., proposed adversarial trained res-net model to showcase the effectiveness of a boundary weighted loss function [147]. Lui et al., improved the performance of segmentation using attention mechanism [148]. Zhang et al., proposed multi-plane fusion architecture for stroke segmentation [149]. Amin et al., employed a high pass filter image to make prominent the in-homogeneity field effect of the MR slicing, and fused it with the input slices [150]. Bui et al., proposed a novel fully automatic Dense Net (adversarially trained) for predicting volumetric probability maps [151]. Xue et al., proposed a multi-modal multi-path convolutional neural network system for automating stroke area segmentation by analyzing brain-behavior relationships, thereby eliminating the need for manual segmentation. Joshi et al., proposed an encoderdecoder CNN (dilated) for an ischemic lesion segmentation task; this helped in preventing data loss which can occur during max-pooling [152].

The penumbra is the area surrounding an ischemic event, which can be salvaged if prompt treatment is received. Delineation of the penumbra in relation to the infarcted core is important for stroke treatment and monitoring the treatment success. Gupta 
et al., proposed a multi-sequence network for the segmentation of ischemic lesions and to differentiate between core and penumbra. Both the core and penumbra sequences are fed into a U-Net type network [153]. Kumar et al., proposed DeepNet framework for ischemic segmentation [154]. Satish et al., presented an automatic method for identification of core and penumbra regions in ischemic lesions using DWI and perfusion-weighted imaging (PWI). In the absence of the availability of more labeled data, the CNN is trained adversarially (i.e., synthesizing images, applying a segmentation loss (cross-entropy)), with aggregated losses from three discriminators (two of which have the relativistic visual Turing test) [155]. Figure 9 shows the stages for lesion segmentation, identification, and classification of stroke regions for deep learning techniques. Table 7 contains a summary of these techniques.

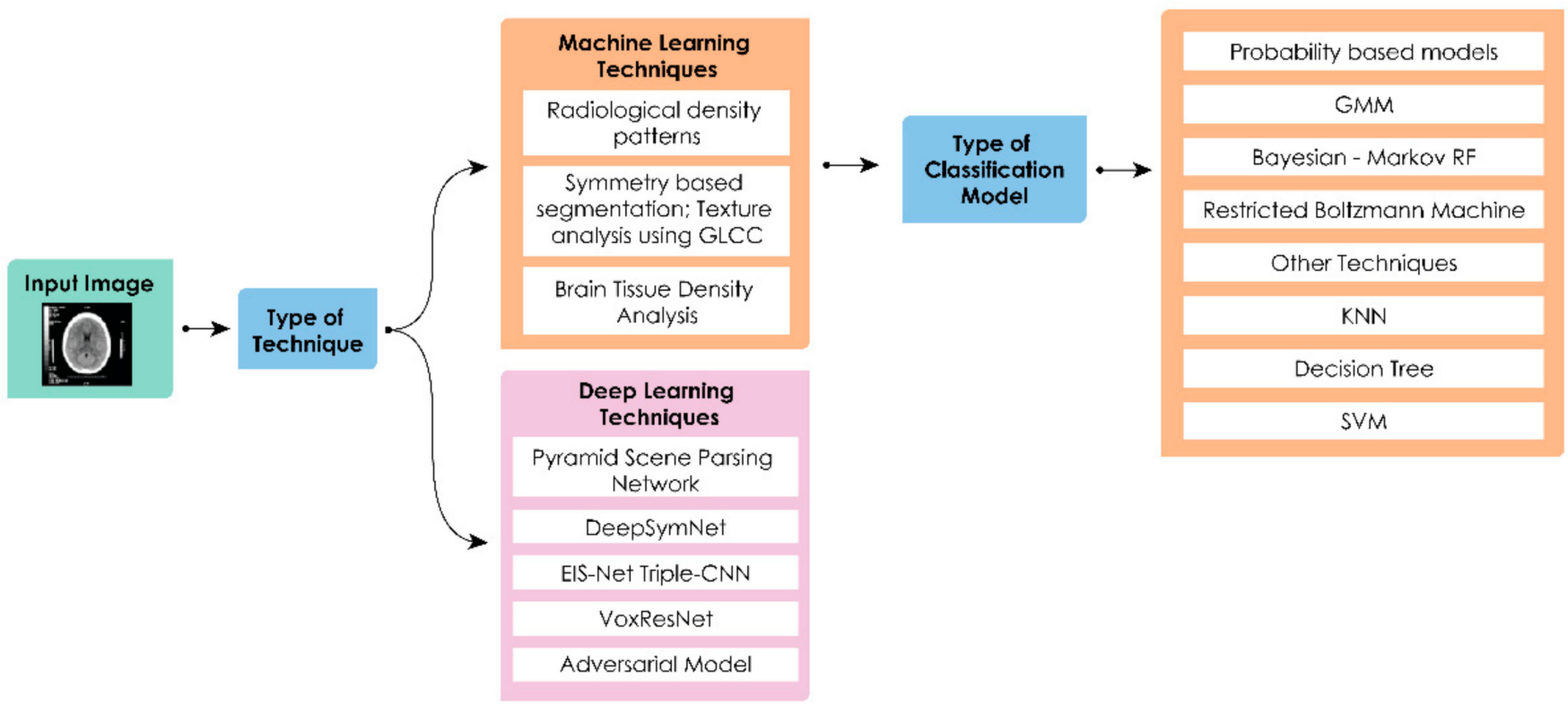

Figure 9. Generalized stages for lesion segmentation, identification, and classification of stroke regions. 
Table 7. Summary of various DL methods applied for IS detection and segmentation.

\begin{tabular}{|c|c|c|c|c|c|}
\hline Articles & Modality & Technique & Loss Function & Outcome & Year \\
\hline Lisowska et al. [99] & NCCT & Bilateral CNN + Atlas & squared hinge loss & $\mathbf{I}_{\text {AUC: }} 0.964$ & 2020 \\
\hline $\begin{array}{c}\text { Abulnaga et al. } \\
{[100]}\end{array}$ & СТР & Pyramid Scene Parsing Network & Focal Loss & $\mathbf{I}_{\mathbf{D C}: 0.54} \pm 0.009$ & 2017 \\
\hline Vargas et al. [102] & CTP & CNN LSTM [Train 356, Validation 40] & & 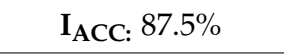 & 2018 \\
\hline Barman et al. [103] & CT A & $\begin{array}{l}\text { DeepSymNet Two identical CNNs } \\
\text { with } 3 \text { Inception module for learning } \\
\text { the low and high level volume 3D } \\
\text { representation common to the two } \\
\text { brain hemispheres. }\end{array}$ & L-1 difference & I $_{\text {AUC: }}: 91.4 \%$ & 2019 \\
\hline $\begin{array}{l}\text { Clèrigues et al. } \\
\text { [104] }\end{array}$ & $\begin{array}{c}\text { CT, } \\
\text { CT-PWI } \\
\text { CBF, CBV\& } \\
\text { MTT }\end{array}$ & $\begin{array}{l}\text { DL based segmentation approach } \\
\text { using 2D patch based for of the acute } \\
\text { stroke lesion core. }\end{array}$ & $\begin{array}{l}\text { To minimize the effects } \\
\text { of class imbalance } \\
\text { Generalized Dice Loss } \\
\text { (GDL) with the cross } \\
\text { entropy loss. }\end{array}$ & $\begin{array}{l}\text { IDSC improvement } \\
\text { of } 4.5 \% \text { over the } \\
\text { baseline [ISLES } \\
2018 \text { ] }\end{array}$ & 2019 \\
\hline $\begin{array}{l}\text { Shinohara et al. } \\
\text { [105] }\end{array}$ & NCCT & $\begin{array}{l}\text { Xception architecture pre-trained on } \\
\text { the ImageNet database }\end{array}$ & classification loss & $\begin{array}{c}\text { ISPEC }_{\text {SP }} 89.7 \% \mathbf{I}_{\mathrm{ACC}} \\
86.5 \%\end{array}$ & 2020 \\
\hline Barros et al. [106] & NCCT & $\begin{array}{l}\text { CNN with two convolutional layers } \\
\text { ( } 256 \text { nodes, } 64 / 128 \text { feature resp.) } \\
\text { followed by } 2 \text { FCN. Each dense layer } \\
\text { has. Max-polling layer with a } 2 \times 2 \\
\text { kernel and a } 2 \times 2 \text { stride. }\end{array}$ & & $\begin{array}{c}\text { Severe } \mathbf{I}_{\mathbf{A C C}}: 0.98 \\
\text { Intermediate } \mathbf{I}_{\mathbf{A C C}}: \\
0.93 \\
\text { Subtle } \mathbf{I}_{\mathbf{A C C}}: 0.66\end{array}$ & 2019 \\
\hline Oman et al. [107] & $\begin{array}{l}\text { CTA, } \\
\text { NCCT }\end{array}$ & 3D CNN & & $\mathbf{I}_{\mathrm{DC}}: 0.61$ & 2019 \\
\hline Hu et al. [108] & 3D MRI & $3 \mathrm{D}$ residual framework & Focal Loss & $\begin{array}{c}\text { BRATS } 2015 \\
\text { IDC: } 0.86 \text { (whole) }\end{array}$ & 2020 \\
\hline Bertels et al. [110] & СТР & Contra Lateral Information CNN & Binary cross-entropy & $\begin{array}{c}\text { I }_{\text {DC: }}: 0.45 \text { [ISLES } \\
2018]\end{array}$ & 2018 \\
\hline Kuang et al. [111] & NCCT & $\begin{array}{l}\text { EIS-Net Triple-CNN with three triple } \\
\text { encoders and one de-coder with } \\
\text { multi-level attention gate modules. }\end{array}$ & $\begin{array}{l}\text { Combination of } \\
\text { weighted binary cross } \\
\text { entropy and } \\
\text { Generalized } \\
\text { Dice-Coefficient. }\end{array}$ & $\begin{array}{l}\text { I ACC: EIS-Net } \\
85.7 \%\end{array}$ & 2021 \\
\hline $\begin{array}{l}\text { Avetisian et al. } \\
\text { [112] }\end{array}$ & NCCT & $\begin{array}{l}\text { Dual Path Network which fusing the } \\
\text { features of Res-Nets and } \\
\text { densely-connected networks }\end{array}$ & Focal Loss & $\mathbf{I}_{\mathbf{D C}}: 0.703$ & 2020 \\
\hline Wang et al. [114] & MRI & 3D RF trained on ISLES dataset & Hybrid loss function & $\begin{array}{c}\mathbf{I}_{\mathbf{D C}}: 0.16 \pm 0.31 \\
\text { [test] }\end{array}$ & 2016 \\
\hline Havaei M [116] & $\begin{array}{l}\mathrm{T} 1, \mathrm{~T} 2, \mathrm{~T} 1 \mathrm{C} \\
\text { and Flair }\end{array}$ & $\begin{array}{l}\text { CNN (two pathways cascaded } \\
\text { architecture) }\end{array}$ & cross-entropy loss & $\begin{array}{l}\text { SISS I I }: 0.69 \\
\text { SPES I I }: 0.85\end{array}$ & 2020 \\
\hline Chen et al. [121] & DWI & CNN & Cross Entropy & I $_{\text {DC }}: 0.67$ [avg] & 2016 \\
\hline Lucas et al. [121] & $\begin{array}{l}\text { FLAIR, } \\
\text { DWI, T1, } \\
\text { and T2 }\end{array}$ & FCNN-MatConvNet & cross-entropy loss & $\mathbf{I}_{\mathrm{DC}}: 0.59$ & 2017 \\
\hline Alex et al. [123] & $\begin{array}{l}\text { T1, T2,T1C } \\
\text { FLAIR }\end{array}$ & Stacked denoising autoencoders & & $\begin{array}{l}\text { High and Low } \\
\text { Grade Glioma }\end{array}$ & 2017 \\
\hline Lucas et al. [125] & MRI & Res-UNets & $\begin{array}{l}\text { Weighted sum of a } \\
\text { classification and soft } \\
\text { QDice metric }\end{array}$ & $\begin{array}{l}33 \% \text { lower } \\
\text { surface distance } \\
\text { than U-Net }\end{array}$ & 2017 \\
\hline
\end{tabular}


Table 7. Cont.

\begin{tabular}{|c|c|c|c|c|c|}
\hline Articles & Modality & Technique & Loss Function & Outcome & Year \\
\hline Liu et al. [128] & MRI & FCN (Res-FCN) & $\begin{array}{l}\text { Customized Loss } \\
\text { Function }\end{array}$ & $\mathbf{I}_{\mathbf{D C}}: 0.645$ & 2018 \\
\hline Zhang et al. [129] & DWI & 3D FC-DenseNet & $\begin{array}{l}\text { Customized Loss } \\
\text { function + Dice Loss } \\
\text { function }\end{array}$ & IDC: 0.79 [Best] & 2018 \\
\hline Chen et al. [130] & 3D MRI & $\begin{array}{l}\text { VoxResNet: Stacked residual modules } \\
\text { with convolutional/de-convolutional } \\
\text { (total } 25 \text { volumetric) }\end{array}$ & $\begin{array}{l}\text { spatial information } \\
\text { loss }\end{array}$ & $\begin{array}{c}\text { I }_{\mathrm{DC}} \\
\text { GM } 86.15 \\
\text { WM } 89.46 \\
\text { CSF } 84.25\end{array}$ & 2018 \\
\hline
\end{tabular}

Two convolutional layers are repeatedly employed,

Li et al. [131] MRI FLAIR each with ReLU and a $2 \times 2$ (max pooling), down-sampling with stride

Dice Loss

MICCAI 2017

IDSC: 0.80

\begin{tabular}{|c|c|c|c|c|c|}
\hline Praveen et al. [132] & $\begin{array}{l}\text { FLAIR, } \\
\text { DWI, T1, } \\
\text { and T2 }\end{array}$ & $\begin{array}{l}\text { Stacked Sparse autoencoder layers } \\
\text { and support vector machine classifier } \\
\text { as the output layer. }\end{array}$ & Mean Squared Loss & $\begin{array}{c}\text { ISLES } 2015 \\
\mathbf{I}_{\mathbf{D C}}: 0.943 \pm 0.057\end{array}$ & 2018 \\
\hline Li et al. [134] & $\begin{array}{c}\mathrm{CT}, \\
\text { DPWI, CBF }\end{array}$ & Deep Residual Dilated U-Net & Cross-entropy loss & $\begin{array}{l}\text { MICCAI } \\
\mathbf{I}_{\text {DC: }} 0.81\end{array}$ & 2018 \\
\hline Luna et al. [135] & MRI & 3D CNN & $\begin{array}{l}\text { normalized categorical } \\
\text { cross entropy loss }\end{array}$ & $\begin{array}{c}\text { MRBrainS18 } \\
\text { Weighted DC } 4.44\end{array}$ & 2019 \\
\hline $\begin{array}{l}\text { Winzeck et al. } \\
\text { [136] }\end{array}$ & MRI & Ensemble Res-CNN: & $\begin{array}{l}\text { Costumed Loss } \\
\text { Function }\end{array}$ & $\mathbf{I}_{\mathrm{DC}}: 82.2 \%$ & 2019 \\
\hline Li et al. [139] & $\begin{array}{l}\mathrm{T} 1, \mathrm{~T} 2, \mathrm{~T} 1 \mathrm{c} \\
\text { and FLAIR }\end{array}$ & $\begin{array}{l}\text { U-Net structure with a new } \\
\text { cross-layer architecture (up skip } \\
\text { connection) and incorporating } \\
\text { inception modules }\end{array}$ & DSC & $\begin{array}{c}\text { [train] } \mathbf{I}_{\mathbf{D C}}: \\
\text { BRATS } 150.89 \\
\text { BRATS } 170.876\end{array}$ & 2018 \\
\hline Malla et al. [140] & MRI & CNN [Deepmedic] & $\begin{array}{l}\text { Dice Similarity } \\
\text { Coefficient }\end{array}$ & $\begin{array}{c}17 \% \text { improved } \mathbf{I}_{\mathbf{D C}} \text { : } \\
\text { over BS }\end{array}$ & 2019 \\
\hline Yang et al. [141] & T1 MRI & $\begin{array}{l}\text { Cross-level fusion with context } \\
\text { (inference) network for stroke lesion } \\
\text { segmentation (chronic) }\end{array}$ & DLF & $\begin{array}{l}\text { ATLAS } \\
\text { I }_{\mathbf{D C}}: 0.58\end{array}$ & 2019 \\
\hline Qi et al. [142] & MRI & $\begin{array}{l}\text { X-Net (a nonlocal operation to } \\
\text { capture long-ranged dependencies) } \\
\text { or the chronic stroke lesion } \\
\text { segmentation }\end{array}$ & DLF & $\begin{array}{l}\text { ATLAS } \\
\text { I }_{\text {DC: }} 0.48\end{array}$ & 2019 \\
\hline Liu et al. [143] & MRI & $\begin{array}{l}\text { multi-kernel DCNN with pixel } \\
\text { dropout }\end{array}$ & DLF & $\begin{array}{c}\text { SPES } \\
\text { I }_{\mathrm{DC}}: 0.79\end{array}$ & 2019 \\
\hline Chin et al. [144] & MRI & Cascaded Networks (U-Net) & & 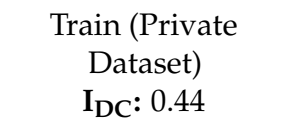 & 2020 \\
\hline Liu et al. [148] & MRI & Attention-based DRANet. & DLF & $\begin{array}{c}\text { (748 Images } \\
\text { Sub-acute) } \\
\text { IDC: } 0.76 \text { (Best) }\end{array}$ & 2016 \\
\hline $\begin{array}{l}\text { ZHANG et al. } \\
\text { [149] }\end{array}$ & DWI & $\begin{array}{l}\text { A triple-branch DSN architecture } \\
\text { with a multi-plane fusion network }\end{array}$ & $\begin{array}{l}\text { Customized Loss } \\
\text { Function }\end{array}$ & $\begin{array}{l}\text { ISLES } 2015 \text { SSIS } \\
\text { IDC: } 0.62\end{array}$ & 2020 \\
\hline Amin et al. [150] & MRI & Auto encoders [segmentation] & & IDC: 0.96 (BRATS) & 2020 \\
\hline Bui et al. [151] & MRI & 3D Dense Net & modified DLF & $\begin{array}{l}\text { MRBrainS18 } \\
\text { I }_{\text {DC }} 0.87\end{array}$ & 2019 \\
\hline
\end{tabular}


Table 7. Cont.

\begin{tabular}{|c|c|c|c|c|c|}
\hline Articles & Modality & Technique & Loss Function & Outcome & Year \\
\hline Joshi et al. [152] & DWI-MRI & Dilated and Transposed CNN & $\begin{array}{l}\text { Binary cross entropy } \\
\text { plus the dice loss }\end{array}$ & $\begin{array}{l}\text { ISLES 2015-2017 } \\
\text { (train } 25000, \\
\text { validation 4000) } \\
\text { I }_{\mathbf{D C}}: 0.85 \\
\text { (validation) and } \\
\text { IJACD }_{\text {JAt } 0.78}\end{array}$ & 2018 \\
\hline Gupta et al. [153] & MRI & $\begin{array}{l}\text { Multi-Sequence Network } \\
\text { architecture: Conv. Layers, Pooling } \\
\text { Layers }(2 \times 2) \text {, Up sampling layers }(2 \\
\times 2) \text {, Dropout Layers, }\end{array}$ & Binary Cross-entropy & $\begin{array}{l}\text { ISLES } 2015 \\
\text { Core Esti } \mathbf{I}_{\text {DC: }} 0.68 \\
\text { Penumbra Esti. } \\
\text { I } \\
\text { IDC } 0.82\end{array}$ & 2019 \\
\hline Kumar et al. [154] & MRI & $\begin{array}{l}\text { Classifier-Segmenter network } \\
\text { (modified UNet for segmentation) }\end{array}$ & $\begin{array}{l}\text { multi-scale loss } \\
\text { function (customized) }\end{array}$ & $\begin{array}{l}\text { ISLES 2017-SPES } \\
\text { dataset I I }: 0.83\end{array}$ & 2020 \\
\hline Satish et al. [155] & DWI, PWI & $\begin{array}{l}\text { Adversarial Architecture: } \\
\text { Encoder-decoder as segmentor. } \\
\text { Discriminators: CNN }\end{array}$ & cross-entropy & $\begin{array}{c}\text { ISLES } 2015 \\
\text { IDC: } 0.82\end{array}$ & 2020 \\
\hline
\end{tabular}

DLF, dice loss function; $\mathbf{I}_{\text {ACC, accuracy; }} \mathbf{I}_{\mathbf{I C C}}$, intra class correlation coefficient; $\mathbf{I}_{\mathbf{P R E C}}$, ischemic precision; $\mathbf{I}_{\mathbf{F S}}$, ischemic FScore; $\mathbf{I}_{\mathbf{S P E C}}$ ischemic specificity; $\mathbf{I}_{\text {SENS }}$, ischemic sensitivity.

\subsubsection{Hemorrhagic Stroke}

Phong et al., compared three types of CNN: LeNet, GoogleLeNet, and Inception ResNet to determine the best method for hemorrhagic stroke detection [156]. Majumdar et al., trained a CNN with improved performance by computing the mean output for rotations of input images [157]. Arbabshirani et al., proposed and tested a predictive DL model capable of detecting ICH [158]. Kuo et al., dealt with a challenge to identify minute and subtle abnormality in a large $3 \mathrm{D}$ volume with superior sensitivity, through an end-toend patch-based FCN model network that performs joint classification and segmentation on CT images [159]. Patel et al., proposed a 3D CNN with a combination of contextual information to detect and segment stroke lesions [160]. Cho et al., proposed a deep learning model that was constructed on two convolutional neural networks and dual FCN to detect bleeding, for classification into five types of ICH for lesion segmentation [161]. The limited hardware poses a problem in computation for deep learning networks, and there exists a tradeoff between hardware and input size (i.e., learning via contextual information). Patel et al., tried a method for the identification of ICH in 3D NCCT. The method combines a CNN and RNN through bidirectional long short-term memory (LSTM) for ICH identification at the image level [162]. Barros et al., proposed and developed CNN for the detection and volumetric segmentation of subarachnoid hemorrhage (SAH) in non-contrast computed tomography (NCCT) [163]. Lee et al., trained a DL model for detection of ICH without backpropagation [164]. Xu et al., introduced the continuous monitoring of health vitals wirelessly through IoT, which is termed 'Health of Things'. The system is capable of classifying CT imagery into uninjured and stroke and thereafter, the segmentation process is carried out via a combination of Masked RNN and ML algorithm [165]. Li et al., proposed a U-net based DL framework to detect and segment hemorrhagic strokes based automatically on CT brain images.

Experiments are conducted to add a symmetrical constraint by using flipped images as input [166]. Arab et al., developed and evaluated an automated DL method with $\mathrm{CNN}$ and deep supervision CNN for precise hematoma (blood clot) segmentation and volumetric quantification in CT images [167]. The combination of CNN with LSTM has yielded good results but shows limited accuracy in performance, as many types use pretrained models. Grewal et al., proposed a RADNET joint CNN and LSTM model which emulates a radiologist for $\mathrm{ICH}$ detection, by performing segmentation at multiple levels of granularity and including a binary classification of intracranial hemorrhage [168]. Burduja et al., proposed a light and efficient network for detecting ICH, consisting of the $\mathrm{CNN}$ and LSTM [169]. A summarized review with details is highlighted in Table 8. 
Table 8. Summary of various methods applied for stroke detection (hemorrhagic).

\begin{tabular}{|c|c|c|c|c|c|}
\hline Modality & Articles & Technique & Loss Function & Outcome & Year \\
\hline $\mathrm{CT}$ & Phong et al. [156] & $\begin{array}{c}\text { LeNet, GoogLeNet, and } \\
\text { Inception-ResNet } \\
\text { Private Dataset of } 1700 \text { records }\end{array}$ & & F1 Score 0.997 (LeNet) & 2017 \\
\hline $\mathrm{CT}$ & Majumdar et al. & $\begin{array}{l}9(3 \times 3) \text { convolutional blocks, }(2 \\
\times 2) \text { max-pooling, BN and ReLU }\end{array}$ & & $\begin{array}{c}81 \% \mathbf{H}_{\text {SENS }} \text { per lesion } \\
98 \% \mathbf{H}_{\text {SPEC }} \text { per case }\end{array}$ & 2018 \\
\hline NCCT & Patel et al. [160] & $\begin{array}{c}\text { CNN with two distinct pathways } \\
\text { integrating contextual } \\
\text { information }\end{array}$ & $\begin{array}{l}\text { categorical cross } \\
\text { entropy }\end{array}$ & $\mathbf{H}_{\text {DC }}: 0.91$ & 2019 \\
\hline $\mathrm{CT}$ & Cho et al. [161] & $\mathrm{FCN}-8 \mathrm{~s}$ & & H $_{\text {ACC }}: 98.28 \%$ & 2019 \\
\hline $\mathrm{CT}$ & Patel et al. [162] & $\mathrm{CNN}$ and $\mathrm{RNN}$ & Binary Cross Entropy & $\mathbf{H}_{\mathrm{ACC}}: 0.87$ & 2019 \\
\hline NCCT & Barros et al. [163] & $\mathrm{CNN}$ & & $\mathbf{H}_{\mathrm{DC}}: 0.63 \pm 0.16$ & 2020 \\
\hline NCCT & Lee et al. [164] & $\mathrm{CNN}$ & & $\mathbf{H}_{\text {AUC: }} 0.903$ & 2020 \\
\hline $\mathrm{CT}$ & Xu et al. [165] & Masked RNN and ML & & $\begin{array}{c}\text { Model[Resnet50+ } \\
\text { MLP+MobileNET] } \\
\text { I }_{\text {ACC: }} \\
\text { EM } 99.89 \text { [Best] }\end{array}$ & 2020 \\
\hline $\mathrm{CT}$ & Li et al. [166] & Pre-trained Dilated UNet & & $\mathbf{H}_{\mathrm{DC}}: 0.8033$ & 2021 \\
\hline NCCT & Arab et al. [167] & $\begin{array}{l}\text { U-Net with deep supervision. } \\
\text { Encoder: Residual block Decoder: } \\
\text { Convl layers }\end{array}$ & $\begin{array}{l}\text { Dice similarity } \\
\text { coefficients }\end{array}$ & $\mathbf{H}_{\mathrm{DC}}: 0.84 \pm 0.06$ & 2020 \\
\hline $\mathrm{CT}$ & Grewal et al. [168] & $\begin{array}{l}\text { Recurrent Attention DenseNet, } \\
\text { bidirectional LSTM layer }\end{array}$ & & $\mathbf{H}_{\mathrm{ACC}}: 0.8182$ & 2018 \\
\hline NCCT & Burduja et al. [169] & CNN \& LSTM & Binary cross-entropy & $\mathbf{H}_{\mathrm{LL}}: 0.04989$ & 2020 \\
\hline
\end{tabular}

ICC, infraclass correlation coefficients; $\mathrm{H}_{\mathrm{DSC}}$, dice similarity coefficient; ASSD, average symmetric surface distance; $\mathrm{H}_{\mathrm{LL}}, \log$ loss; $\mathrm{H}_{\mathrm{SENS}}$, Sensitivity, H $_{\text {SPEC: }}$ Specificity.

\subsubsection{Combined Stroke}

Pereira et al., present a model for stroke detection in CT using CNN optimized by PSO; the shallower network obtained better accuracy than the deeper version [170]. Marbun et al., employed $\mathrm{CNN}$ with proper preprocessing of images (gray scaling, histogram equalization etc.) for classification of type of stroke [171]. Carlos CMD et al., presented an IoT enabled framework with $\mathrm{CNN}$ as the main classifier to identify a healthy or a stroke affected brain from CT images [172]. Kunag et al., proposed to segment ischemic and hemorrhagic infarct simultaneously using a U-Net based architecture. The input is divided into four disjoint regions and CNN was employed to generate probability maps for ischemic, hemorrhagic, and other infarcts [173]. Xuea et al., used multi-modal MRI for classification of types of strokes [174].

\section{Discussion}

\subsection{Non-ML/DL Based Techniques}

Several papers were surveyed under this topic to understand various approaches and techniques. It was found that all of these basically fall under several major categories: region growth, texture extraction (linear, non-linear, and spatial and frequency domain), enhancement and analysis and contour based. Time complexity was largely governed by modality type in consideration with the degree of imaging modals. Although many algorithms have automated the processes, much of this work still requires manual intervention and supervision. Work towards consideration of data encompassing various other parameters such as age and essential clinical parameters could be helpful in making a more comprehensive analysis. 


\subsection{Based Techniques}

Publications in this domain may be classified into three major types: (1) classification based on discriminants such as texture, brain tissue density, contour-based analysis; (2) purely probabilistic types; and (3) hybrid types, where the results are refined by other methods. Although the former is efficient in terms of reducing time complexity and processing, they suffer from the need of specialist intervention to indicate region and to affirm the stroke type. Furthermore, for effective demarcation there is a need for optimal radiation attenuation, which poses as considerable challenge. Most of the texture-based algorithms have the drawback of missing barely visible lesions due to subtle intensity differences, scan image quality, and intensity inhomogeneity, which causes high false positive rates. A system with medical expert assistance to aid in the technical detection could prove helpful for network training [175]. It is therefore expected that future research directions will include the development of intensity resilient algorithms which perhaps could be coupled with better image enhancement techniques.

\subsection{Based Techniques}

Emergence of deep learning has established a new paradigm in the domain of stroke detection. Many papers have shown remarkable progress in terms of time, accuracy, and adaptability. Their ability to customize feature importance and to identify discriminating features including the presence of hyperdense vessels (sign of a large vessel occlusion), and disturbed symmetry of vascular and brain tissue textures, has been primarily used for prognosis and automatic detection of lesions. Architectures such as CNN with 3D kernels have been extensively explored with many modifications in loss functions (specialized focus) and adaptations to novel model architectures (U-Nets, ResNets, etc.) have led to improved efficiency. However, CNN architectures are limited in accuracy when segmenting ischemic stroke areas, and their heterogeneity in location, shape, size, image intensity, and texture are the main reasons for their reduced level of efficacy, especially in this imaging modality. Although such techniques rely on self-extraction of features, it was found that many yielded better results after providing additional information including the use of atlas coordinates to show dependency. In CNN architectures, the kernel size determines the ROI, thereby affecting diagnostic performance. Smaller kernels lead to missing specific regions and bigger kernels lead to heavy parametrization. Hence, a tradeoff is imperative. Most of the considered datasets seem to be highly imbalanced leading to overfitting; this could be solved by incorporating precision or recall based upon an objective function with data fusion [175]. Moreover, data augmentation techniques and generation of synthetic data using GAN may need to be utilized. We found a lesser number of papers dedicated to the delineation of core and penumbra regions separately. Hence, further research should be extended in that direction.

\subsection{Preferred Choice of Diagnostic Imaging}

There is no single globally preferred choice in modality either for lesion separation or stroke detection; all have their benefits and are specific to certain tasks. In our study we found that there is an urgent need for better imaging analysis technology to improve inference, and a need for advancements in imaging techniques, as detection of incomplete infarction in the acute stroke setting on MRI or CT is currently not feasible (since current CT and MRI modalities are subjected to partial volume averaging as well as very limited spatial and tissue resolution). Yet in practical scenarios, accessibility must be the primary concern for such applications. As CT is readily accessible, affordable, and less contraindicated, especially for hemorrhagic stroke analysis, it is seen as a natural choice for implementation [175]. In most of the analyses we found that CT is useful in many applications, providing acceptable results and often the best results. We noticed many papers which employed raw $\mathrm{CT}$ as their base modality. In advanced cases, perfusion $\mathrm{CT}$ addresses many critical unknowns in the acute stroke triage, and it is apt in the task 
of delineating the operational penumbra from the infarct core. In application of acute intracranial ischemic/hemorrhage NCCT this was seen as extensively useful.

More work should be carried out in the advancement of CT or its variants in application of networks to explore the core as a potential area to improve stroke analysis. We do not claim that $\mathrm{CT}$ is better than MRI for this purpose, but from a practical standpoint there is reason to enhance the processing of $\mathrm{CT}$ data for preliminary diagnostics and prior to the use of higher advanced modalities in following stages.

\subsection{Time Complexity}

Time complexity is a measure of the time consumption of algorithms as a function of inputs, preferably measured for the worst-case scenario to set the upper limit [176]. Although it is an important metric for gauging algorithms, it is tenuous when used for complex domains such as segmentation, detection, and prediction of intricate regions, as they are often an amalgamation of many techniques working either sequentially or in parallel. In this review, few studies have reported the time taken for processing per input or relative improvement in terms of time while also providing a detailed analysis of their algorithm in terms of time and space complexity. Including a section on "computational and space complexity" of their model can be one of the evaluation parameters used to assess the performance of the model.

\subsection{Prognosis}

The ultimate goal of ischemic stroke treatment is to recanalize an occluded vessel and enable damage control. It is important for timely and precise decision-making, which ultimately affects patient outcomes. This study attended to the detail of stroke prognosis, plausibility of treatment options, and relevance. We studied many research papers which performed and successfully executed analysis to aid in diagnosis and prognosis. A summary is illustrated in Table 9. There were many dimensions that investigators used such as prediction of infarct growth over time and predicting the functional outcome of ischemic stroke patients, but these were limited by insufficient data, lack of manual supervision, and massive size. As many studies have pointed out, the most probable reason behind stroke is older age, lifestyle, low level of physical activity, unhealthy diet, hypertension, smoking, and diabetes mellitus, which makes the prognosis based on a specific source difficult. In this regard, we wish to encourage the research community to explore the areas of personalized diagnostics with many sources of consideration. Deep learning models utilizing image features coupled with other information about the patient could yield better and more accurate results. The capturing of data can be carried out via a home medical teleport, or from nearby health centers, and then transmitted to cloud-based models via mobile apps, even in remote regions of the world with lesser available services. Cloud-based models trained and maintained using federated learning are potentially much more reliable than current methods and could revolutionize this field. 
Table 9. Summary of different methods applied for prognosis of strokes.

\begin{tabular}{|c|c|c|c|c|}
\hline Articles & Modality & Technique & Prediction & Year \\
\hline Rebouças et al. [51] & CT & $\begin{array}{l}\text { Feature extraction based on density patterns } \\
\text { (radiological) and classification of strokes through } \\
\text { kNN, SVM, MLP, OPF and Bayesian classifiers }\end{array}$ & $\begin{array}{l}\text { Identify \& classify the } \\
\text { occurrence of strokes (extent } \\
\text { and severity). }\end{array}$ & 2017 \\
\hline Robben et al. [84] & СТP & Modifed DeepMedic & Final infarct volume & 2019 \\
\hline Bentley et al. [97] & CT & SVM with an $\mathbf{H}_{\mathrm{AUC}}$ : 0.744 & $\begin{array}{l}\text { Predict symptomatic } \\
\text { intracranial hemorrhage }\end{array}$ & 2014 \\
\hline Stier et al. [117] & Tmax MRI & $\begin{array}{l}\text { CNN with } 2 \text { Conv layers, } 26 \times 6 \text { pooling layers, } \\
\text { trained with } 100 \text { epochs for Binary prediction }\end{array}$ & Tissue Fate Features in AIS & 2016 \\
\hline Choi et al. [119] & MRI & $\begin{array}{l}\text { Lesion outcome prediction-3D Res } \\
\text { U-Net-CNN Clinical outcome } \\
\text { prediction-CNN-Log Regression }\end{array}$ & $\begin{array}{l}\text { Automated prognosis for } \\
\text { post-treatment ischemic stroke }\end{array}$ & 2016 \\
\hline Chen et al. [121] & CT & $\mathrm{CNN}$ & $\begin{array}{c}\text { Early stroke detection } \\
\text { (ischemic) system with CNN }\end{array}$ & 2017 \\
\hline Lucas et al. [122] & CT & $\begin{array}{l}\text { 3D U-net appended with Convolutional } \\
\text { auto-encoder }\end{array}$ & & 2018 \\
\hline Lucas et al. [125] & CT & 3D UNets & $\begin{array}{l}\text { Predict Ischemic Stroke } \\
\text { Growth }\end{array}$ & 2018 \\
\hline Bento et al. [126] & & SVM I ICC: $97.5 \%$ & $\begin{array}{c}\text { Early identification of } \\
\text { Carotidartery Atherosclerosis }\end{array}$ & 2019 \\
\hline Song et al. [127] & & GAN I DC: $_{0.624}$ & $\begin{array}{l}\text { Prediction of perfusion } \\
\text { parameters }\end{array}$ & 2019 \\
\hline $\begin{array}{l}\text { Giacalone et al. } \\
\text { [124] }\end{array}$ & & SVM IPRES: 95\% & Final lesion prediction & 2018 \\
\hline $\begin{array}{l}\text { Arbabshirani et al. } \\
\text { [158] }\end{array}$ & CT & DCNN HAUC: 0.846 & $\begin{array}{l}\text { Detecting of ICH based on } \\
\text { clinical database of brain CT } \\
\text { images }\end{array}$ & \\
\hline
\end{tabular}

\section{Challenges and Future Directions}

While surveying the field, we encountered many approaches, applications, and techniques based on various datasets, and it was difficult to evaluate them all in generality. Many methods claimed to be fully automated yet relied on human assistance/interaction for parameter initialization. A fully automated process would require a powerful intelligent system which can adapt and customize based on patient condition/severity of symptoms and would avail a host of opportunities regarding the prospects of artificial intelligence in this area. A remarkable progress in terms of segmentation has already been carried out, yet the task of specifically locating the penumbra and core is still to be explored and refined. Concerning identification of the penumbra, more work is needed as this would help to rejuvenate it faster. In stroke detection, we found less work in identification of its sub-classes and a lack of research on the dynamic evolution of stroke as time progresses. A heterogeneous dataset containing images across the regions and countries with different conditions must be developed for better research and more clarity on their impact.

Future research can be directed in several ways:

1. IoT based personalized AI: AI being the main protagonist of Industry 4.0, having farreaching implications, especially in healthcare. Hyper personalization of healthcare could provide tailor-made diagnostics and would vastly improve early detection of disease.

2. Creation of a large hetero public database: The dataset that we have addressed consists of few images for train and test, with regard to particular domain or region. A larger public dataset would assist to better cover major areas. 
3. Remote patient monitoring through federated learning: Figure 10 shows a prototype for remote patient monitoring with cloud-based AI Models. Wearable modes with continuous monitoring of biomarkers with easy transfer of meta-data to cloud through phones for collective learning and personalized prediction would be helpful. These could act as a digital expert to assist in patient diagnosis and prognosis.

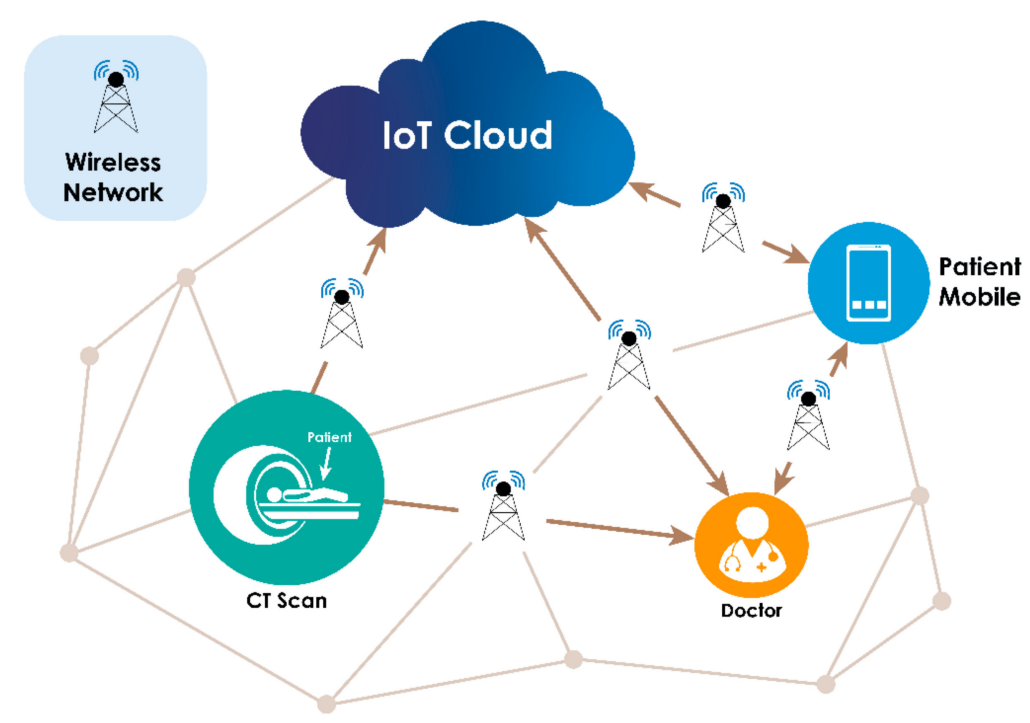

Figure 10. Prototype model for remote patient monitoring with cloud-based AI Model.

\section{Conclusions}

In this study we reviewed the status, trends, and future directions in stroke detection and segmentation. It is clear that a rapid, adaptable process facilitating timely neuroimaging analysis is imperative in stroke management. This is due to the fact that neuroimaging has a prime role in the diagnosis and optimal management of different types of strokes. Due to recent advancements in neuroimaging, AI, and computation power, the development of automated diagnostic tools is clearly within reach. Advances in this field and translation into clinical practice will result in reduced patient morbidity and mortality.

Based on the findings of this systematic literature review, we make a number of suggestions for how the performance of automated diagnostic tools can be improved and a more comprehensive automated system can be built. Firstly, segmentation techniques could be automated and personalized for individual patients, allowing translation of clinical research into clinical diagnostic practice. Secondly, instead of training the systems on specific datasets, a more heterogeneous dataset could be considered. This would help the models to comprehensively learn all cases, both regionally and country-wide. Thirdly, besides the prospects mentioned on the different stroke stages in Section 5, a fully automated segmentation system with deeper networks for stroke area segmentation (especially in sub-acute and chronic strokes) could be built in for future paradigms. Fourthly, as there have been recent attempts to predict infarcts and the extent of the penumbra, more research should be focused on these strategies, in order to devise and combine prognostic tools for strokes, such as the degree of infraction, into stroke management algorithms. Fifthly, GANs could certainly prove helpful in generating synthetic datasets in cases of data scarcity, class imbalance and cases when the cost of obtaining labelled data is huge. However, care must be taken to keep original distribution unperturbed and that new data doesn't create any bias in the decision-making process. Sixthly, segmentation models greatly depend on quality of image quality, acquisition and the reconstruction parameters of the modality. Small changes in these parameters can lead to a substantial deviation in the output in model output [177]. It could be better to have proper standards for parameters can which could potentially prevent this and help in improving the reproducibility of the results. Lastly, it would be ideal to explore the possibility of remote patient monitoring, to enhance 
equity of access to excellent stroke management in the most cost-effective and acceptable manner, and also boost the prediction of stroke, with the potential to prevent this disabling condition arising in the first place, thereby trans-forming patient outcome. Perhaps in the future, the accuracy of stroke prediction via metadata analysis will be an important criterion to evaluate stroke segmentation results.

Author Contributions: Data curation, writing-review and editing, original draft preparation, M.A.I., U.R. and A.G.; conceptualization and supervision, U.R.; formal analysis, clinical formulation, A.H., G.R.M., P.B., E.E.P., K.H.C. and W.Y.C.; visualization, M.A.I. and Y.C.; supervision, E.J.C. and U.R.A. All authors have read and agreed to the published version of the manuscript.

Funding: This research received no external funding.

Institutional Review Board Statement: Not applicable.

Informed Consent Statement: Not applicable.

Conflicts of Interest: The authors declare no conflict of interest.

\section{References}

1. Virani, S.S.; Alonso, A.; Aparicio, H.J.; Benjamin, E.J.; Bittencourt, M.S.; Callaway, C.W.; Carson, A.P.; Chamberlain, A.M.; Cheng, S.; Delling, F.N.; et al. Heart Disease and Stroke Statistics-2021 Update: A Report from the American Heart Association. Available online: https:/ / www.heart.org/-/media/PHD-Files-2/Science-News/2/2021-Heart-and-Stroke-Stat-Update/2021 _Stat_Update_factsheet_Global_Burden_of_Disease.pdf (accessed on 27 January 2021). [CrossRef]

2. GBD 2016 Stroke Collaborators. Global, regional, and national burden of stroke, 1990-2016: A systematic analysis for the Global Burden of Disease Study 2016. Lancet 2019, 18, 439-458. [CrossRef]

3. Feigin, V.L.; Norrving, B.; Mensah, G.A. Global Burden of Stroke. Circ. Res. 2017, 120, 439-448. [CrossRef] [PubMed]

4. O'Donnell, M.J.; Xavier, D.; Liu, L.; Zhang, H.; Chin, S.L.; Rao-Melacini, P.; Rangarajan, S.; Islam, S.; Pais, P.; McQueen, M.J.; et al. Risk factors for ischaemic and intracerebral haemorrhagic stroke in 22 countries (the INTERSTROKE study): A case-control study. Lancet 2010, 376, 112-123. [CrossRef]

5. Campbell, B.C.V.; De Silva, D.A.; MacLeod, M.R.; Coutts, S.B.; Schwamm, L.H.; Davis, S.M.; Donnan, G.A. Ischaemic stroke. Nat. Rev. Dis. Primers 2019, 5, 70. [CrossRef]

6. Switzer, A.J.; Hess, D.C.; Nichols, F.T.; Adams, R.J. Pathophysiology and treatment of stroke in sickle-cell disease: Present and future. Lancet Neurol. 2006, 5, 501-512. [CrossRef]

7. Krishnamurthi, R.V.; Feigin, V.L.; Forouzanfar, M.H.; Mensah, A.G.; Connor, M.; Bennett, A.D.; Moran, E.A.; Sacco, R.L.; Anderson, L.M.; Truelsen, T.; et al. Global and regional burden of first-ever ischaemic and haemorrhagic stroke during 1990-2010: Findings from the Global Burden of Disease Study. Lancet Glob. Health 2013, 1, e259-e281. [CrossRef]

8. Sagar, R.; Dandona, R.; Gururaj, G.; Dhaliwal, R.S.; Singh, A.; Ferrari, A.; Dua, T.; Ganguli, A.; Varghese, M.; Chakma, J.K.; et al. The burden of neurological disorders across the states of India: The Global Burden of Disease Study 1990-2019. Lancet Psychiatry 2020, 7, 148-161. [CrossRef]

9. Kamalakannan, S.; Gudlavalleti, A.; Gudlavalleti, V.S.M.; Goenka, S.; Kuper, H. Incidence \& prevalence of stroke in India: A systematic review. Indian J. Med. Res. 2017, 146, 175-185. [CrossRef]

10. Donkor, E.S. Stroke in the21st Century: A Snapshot of the Burden, Epidemiology, and Quality of Life. Stroke Res. Treat. 2018, 2018, 3238165. [CrossRef]

11. Saver, J.L. Penumbral salvage and thrombolysis outcome: A drop of brain, a week of life. Brain 2017, 140, 519-522. [CrossRef]

12. Meretoja, A.; Keshtkaran, M.; Tatlisumak, T.; Donnan, G.A.; Churilov, L. Endovascular therapy for ischemic stroke: Save a minute-save a week. Neurology 2017, 88, 2123-2127. [CrossRef]

13. Powers, W.J.; Rabinstein, A.A.; Ackerson, T.; Adeoye, O.M.; Bambakidis, N.C.; Becker, K.; Biller, J.; Brown, M.; Demaerschalk, B.M.; Hoh, B.; et al. 2018 Guidelines for the Early Management of Patients with Acute Ischemic Stroke: A Guideline for Healthcare Professionals from the American Heart Association/American Stroke Association. Stroke 2018, 49, e46-e99. [CrossRef]

14. Lindsay, P.; Furie, K.L.; Davis, S.M.; Donnan, G.; Norrving, B. World Stroke Organization Global Stroke Services Guidelines and Action Plan. Int. J. Stroke 2014, 9, 4-13. [CrossRef]

15. Brazzelli, M.; Sandercock, P.A.; Chappell, F.M.; Celani, M.G.; Righetti, E.; Arestis, N.; Wardlaw, J.M.; Deeks, J.J. Magnetic resonance imaging versus computed tomography for detection of acute vascular lesions in patients presenting with stroke symptoms. Cochrane Database Syst. Rev. 2009, 4, CD007424. [CrossRef]

16. Clinical Guidelines for Stroke Management. Available online: https://informme.org.au/en/Guidelines/Clinical-Guidelines-forStroke-Management (accessed on 3 October 2021).

17. Bivard, A.; Churilov, L.; Parsons, M. Artificial intelligence for decision support in acute stroke-Current roles and potential. Nat. Rev. Neurol. 2020, 16, 575-585. [CrossRef]

18. Rowley, H.; Vagal, A. Stroke and Stroke Mimics: Diagnosis and Treatment. In Diseases of the Brain, Head and Neck, Spine 2020-2023: Diagnostic Imaging; IDKD Springer Series; Springer: Berlin/Heidelberg, Germany, 2020; pp. 25-36. 
19. Allen, M.L.; Anton, N.; Hasso, J.; Handwerker, J.; Hamed, F. Sequence-specific MR Imaging Findings that Are Useful in Dating Ischemic Stroke. RadioGraphics 2012, 32, 1285-1297. [CrossRef]

20. Moreau, F.; Asdaghi, N.; Modi, J.; Goyal, M.; Coutts, S.B. Magnetic Resonance Imaging versus Computed Tomography in Transient Ischemic Attack and Minor Stroke: The More You See the More You Know. Cerebrovasc. Dis. Extra 2013, 3, $130-136$. [CrossRef]

21. National Institutes of Health. MRI More Sensitive Than CT in Diagnosing Most Common Form of Acute Stroke, Finds NIH Study. Available online: https: / / www.nih.gov/news-events/news-releases/mri-more-sensitive-ct-diagnosing-most-common-formacute-stroke-finds-nih-study (accessed on 4 October 2021).

22. Hopyan, J.; Ciarallo, A.; Dowlatshahi, D.; Howard, P.; John, V.; Yeung, R.; Zhang, L.; Kim, J.; Macfarlane, G.; Lee, T.-Y.; et al. Certainty of Stroke Diagnosis: Incremental Benefit with CT Perfusion over Noncontrast CT and CT Angiography. Radiology 2010, 255, 142-153. [CrossRef]

23. Karthik, R.; Menaka, R.; Johnson, A.; Anand, S. Neuroimaging and deep learning for brain stroke detection-A review of recent advancements and future prospects. Comput. Methods Programs Biomed. 2020, 197, 105728. [CrossRef]

24. Karthik, R.; Menaka, R. Computer-aided detection and characterization of stroke lesion-A short review on the current state-of-the art methods. Imaging Sci. J. 2017, 66, 836-843. [CrossRef]

25. Zhang, Y.; Liu, S.; Li, C.; Wang, J. Review: Application of Deep Learning Method on Ischemic Stroke Lesion Segmentation. J. Shanghai Jiaotong Univ. 2021, 1-13. [CrossRef]

26. Sirsat, M.S.; Fermé, E.; Câmara, J. Machine Learning for Brain Stroke: A Review. J. Stroke Cerebrovasc. Dis. 2020, 29 , 105162. [CrossRef]

27. Wadhwa, A.; Bhardwaj, A.; Verma, V.S. A review on brain tumor segmentation of MRI images. Magn. Reson. Imaging 2019, 61, 247-259. [CrossRef]

28. Magnetic Resonance Imaging (MRI) of the Brain and Spine: Basics. Available online: https://case.edu/med/neurology/NR/ MRI\%20Basics.htm (accessed on 4 October 2021).

29. Niknejad, M.; Bell, D. Apparent Diffusion Coefficient. Available online: https://radiopaedia.org/articles/apparent-diffusioncoefficient-1 (accessed on 4 October 2021).

30. Chalela, J.A.; Kidwell, C.S.; Nentwich, L.M.; Luby, M.; Butman, J.A.; Demchuk, A.M.; Hill, M.D.; Patronas, N.; Latour, L.; Warach, $\mathrm{S}$. Magnetic resonance imaging and computer tomography in emergency assessment of patients with suspected acute stroke: A prospective comparison. Lancet 2007, 369, 293-298. [CrossRef]

31. Mainali, S.; Wahba, M.; Elijovich, L. Detection of Early Ischemic Changes in Noncontrast CT Head Improved with "Stroke Windows". ISRN Neurosci. 2014, 2014, 654980. [CrossRef]

32. Tee, Y.J.; Gaillard, F. Ischemic Stroke. Available online: https:/ / radiopaedia.org/articles/ischaemic-stroke (accessed on 4 October 2021).

33. Macellari, F.; Paciaroni, M.; Agnelli, G.; Caso, V. Neuroimaging in Intracerebral Hemorrhage. Stroke 2014, 45, 903-908. [CrossRef]

34. Smith, E.E.; Rosand, J.; Greenberg, S.M. Imaging of Hemorrhagic Stroke. Magn. Reson. Imaging Clin. N. Am. 2006, 14, 127-140. [CrossRef] [PubMed]

35. Kang, B.K.; Na, D.G.; Ryoo, J.W.; Byun, H.S.; Roh, H.G.; Pyeun, Y.S. Diffusion-Weighted MR Imaging of Intracerebral Hemorrhage. Korean J. Radiol. 2001, 2, 183-191. [CrossRef] [PubMed]

36. French, B.R.; Boddepalli, R.S.; Govindarajan, R. Acute Ischemic Stroke: Current Status and Future Directions. Mo. Med. 2016, 113, 480-486. [PubMed]

37. Lebedev, G.; Meshcheryakova, A.; Kurenkov, V.; Kluganov, V.; Sologubov, A.; Logacheva, N.; Radzievskiy, G.; Klimenko, H. Application of artificial intelligence methods to recognize pathologies on photographs of fundus. Procedia Comput. Sci. 2020, 176, 1823-1828. [CrossRef]

38. Tang, F.-H.; Ng, K.S.; Chow, H.K.D. An image feature approach for computer-aided detection of ischemic stroke. Comput. Biol. Med. 2011, 41, 529-536. [CrossRef]

39. Sajjadi, M.; Amirfattahi, R.; Ahmadzadeh, M.R.; Saghafi, M.A. A new filter bank algorithm for enhancement of early signs of ischemic stroke in brain CT images. In Proceedings of the 2011 IEEE International Conference on Signal and Image Processing Applications (ICSIPA), Kuala Lumpur, Malaysia, 16-18 November 2011; pp. 384-389. [CrossRef]

40. Nowinski, W.L.; Gupta, V.; Qian, G.; He, J.; Poh, L.E.; Ambrosius, W.; Chrzan, R.M.; Polonara, G.; Mazzoni, C.; Mol, M.; et al. Automatic Detection, Localization, and Volume Estimation of Ischemic Infarcts in Noncontrast Computed Tomographic Scans. Investig. Radiol. 2013, 48, 661-670. [CrossRef]

41. Filho, P.P.R.; Sarmento, R.M.; Holanda, G.B.; Lima, D.D.A. New approach to detect and classify stroke in skull CT images via analysis of brain tissue densities. Comput. Methods Programs Biomed. 2017, 148, 27-43. [CrossRef]

42. Flottmann, F.; Broocks, G.; Faizy, T.D.; Ernst, M.; Forkert, N.D.; Grosser, M.; Thomalla, G.; Siemonsen, S.; Fiehler, J.; Kemmling, A. CT-perfusion stroke imaging: A threshold free probabilistic approach to predict infarct volume compared to traditional ischemic thresholds. Sci. Rep. 2017, 7, 6679. [CrossRef]

43. Sakai, Y.; Delman, B.; Fifi, J.T.; Tuhrim, S.; Wheelwright, D.; Doshi, A.H.; Mocco, J.; Nael, K. Estimation of Ischemic Core Volume Using Computed Tomographic Perfusion. Stroke 2018, 49, 2345-2352. [CrossRef]

44. Lo, C.-M.; Hung, P.-H.; Hsieh, K.L.-C. Computer-Aided Detection of Hyperacute Stroke Based on Relative Radiomic Patterns in Computed Tomography. Appl. Sci. 2019, 9, 1668. [CrossRef] 
45. Kamalian, S.; Kamalian, S.; Konstas, A.A.; Maas, M.B.; Payabvash, S.; Pomerantz, S.R.; Schaefer, P.W.; Furie, K.L.; González, R.G.; Lev, M.H. CT Perfusion Mean Transit Time Maps Optimally Distinguish Benign Oligemia from True "At-Risk" Ischemic Penumbra, but Thresholds Vary by Postprocessing Technique. Am. J. Neuroradiol. 2011, 33, 545-549. [CrossRef]

46. Kheradmand, A.; Fisher, M.; Paydarfar, D. Ischemic Stroke in Evolution: Predictive Value of Perfusion Computed Tomography. J. Stroke Cerebrovasc. Dis. 2014, 23, 836-843. [CrossRef]

47. Kawiorski, M.M.; Vicente, A.; Lourido, D.; Muriel, A.; Fandiño, E.; Méndez, J.C.; Sánchez-González, V.; Aguado, A.; ÁlvarezVelasco, R.; De Leciñana, M.A. Good Clinical and Radiological Correlation from Standard Perfusion Computed Tomography Accurately Identifies Salvageable Tissue in Ischemic Stroke. J. Stroke Cerebrovasc. Dis. 2016, 25, 1062-1069. [CrossRef]

48. Bhadauria, H.S.; Dewal, M.L. Intracranial hemorrhage detection using spatial fuzzy c-mean and region-based active contour on brain CT imaging. Signal Image Video Process. 2012, 8, 357-364. [CrossRef]

49. De Haan, B.; Clas, P.; Juenger, H.; Wilke, M.; Karnath, H.-O. Fast semi-automated lesion demarcation in stroke. NeuroImage Clin. 2015, 9, 69-74. [CrossRef]

50. Yahiaoui, A.F.Z.; Bessaid, A. Segmentation of ischemic stroke area from CT brain images. In Proceedings of the 2016 International Symposium on Signal, Image, Video and Communications (ISIVC), Tunis, Tunisia, 21-23 November 2016; pp. 13-17.

51. Reboucas, E.D.S.; Braga, A.M.; Sarmento, R.M.; Marques, R.; Filho, P.P.R. Level Set Based on Brain Radiological Densities for Stroke Segmentation in CT Images. In Proceedings of the 2017 IEEE 30th International Symposium on Computer-Based Medical Systems (CBMS), Thessaloniki, Greece, 22-24 June 2017; pp. 391-396.

52. Kumar, I.; Bhatt, C.; Singh, K.U. Entropy based automatic unsupervised brain intracranial hemorrhage segmentation using CT images. J. King Saud Univ. Comput. Inf. Sci. 2020, in press. [CrossRef]

53. Vasconcelos, F.F.; Sarmento, R.M.; Filho, P.P.R.; de Albuquerque, V.H.C. Artificial intelligence techniques empowered edge-cloud architecture for brain CT image analysis. Eng. Appl. Artif. Intell. 2020, 91, 103585. [CrossRef]

54. Nabizadeh, N.; John, N.; Wright, C. Histogram-based gravitational optimization algorithm on single MR modality for automatic brain lesion detection and segmentation. Expert Syst. Appl. 2014, 41, 7820-7836. [CrossRef]

55. Ghosh, N.; Sun, Y.; Bhanu, B.; Ashwal, S.; Obenaus, A. Automated detection of brain abnormalities in neonatal hypoxia ischemic injury from MR images. Med. Image Anal. 2014, 18, 1059-1069. [CrossRef]

56. Cauley, K.A.; Mongelluzzo, G.J.; Fielden, S.W. Automated Estimation of Acute Infarct Volume from Noncontrast Head CT Using Image Intensity Inhomogeneity Correction. Int. J. Biomed. Imaging 2019, 2019, 1720270. [CrossRef]

57. Ledig, C.; Heckemann, R.; Hammers, A.; Lopez, J.C.; Newcombe, V.; Makropoulos, A.; Lötjönen, J.; Menon, D.K.; Rueckert, D. Robust whole-brain segmentation: Application to traumatic brain injury. Med. Image Anal. 2015, 21, 40-58. [CrossRef]

58. Nazari-Farsani, S.; Nyman, M.; Karjalainen, T.; Bucci, M.; Isojärvi, J.; Nummenmaa, L. Automated segmentation of acute stroke lesions using a data-driven anomaly detection on diffusion weighted MRI. J. Neurosci. Methods 2020, 333, 108575. [CrossRef]

59. Moeskops, P.; Viergever, M.A.; Mendrik, A.M.; De Vries, L.S.; Benders, M.J.N.L.; Isgum, I. Automatic Segmentation of MR Brain Images with a Convolutional Neural Network. IEEE Trans. Med. Imaging 2016, 35, 1252-1261. [CrossRef]

60. Puonti, O.; van Leemput, K. Simultaneous whole-brain segmentation and white matter lesion detection using contrast-adaptive probabilistic models. In Proceedings of the Brainles 2015 MICCAI Workshop, Munich, Germany, 5 October 2015; pp. 12-19.

61. Si, T.; De, A.; Bhattacharjee, A.K. Segmentation of Brain MRI Using Wavelet Transform and Grammatical Bee Colony. J. Circuits Syst. Comput. 2018, 27, 1850108. [CrossRef]

62. Haeck, T.; Maes, F.; Suetens, P. ISLES Challenge 2015: Automated Model-Based Segmentation of Ischemic Stroke in MR Images. In BrainLes 2015, Proceedings of the First International Workshop, Held in Conjunction with MICCAI 2015, Munich, Germany, 5 October 2015; Lecture Notes in Computer Science; Springer: Berlin/Heidelberg, Germany, 2016; pp. 246-253.

63. Ji, Z.; Xia, Y.; Zheng, Y. Robust generative asymmetric GMM for brain MR image segmentation. Comput. Methods Programs Biomed. 2017, 151, 123-138. [CrossRef] [PubMed]

64. Kamnitsas, K.; Ledig, C.; Newcombe, V.; Simpson, J.P.; Kane, A.D.; Menon, D.K.; Rueckert, D.; Glocker, B. Efficient multi-scale 3D CNN with fully connected CRF for accurate brain lesion segmentation. Med. Image Anal. 2017, 36, 61-78. [CrossRef] [PubMed]

65. Rajini, N.H.; Bhavani, R. Computer aided detection of ischemic stroke using segmentation and texture features. Measurement 2013, 46, 1865-1874. [CrossRef]

66. Chen, Y.; Dhar, R.; Heitsch, L.; Ford, A.; Fernandez-Cade, I.; Carrera, C.; Montaner, J.; Lin, W.; Shen, D.; An, H.; et al. Automated quantification of cerebral edema following hemispheric infarction: Application of a machine-learning algorithm to evaluate CSF shifts on serial head CTs. NeuroImage Clin. 2016, 12, 673-680. [CrossRef]

67. Dhar, R.; Chen, Y.; An, H.; Lee, J.-M. Application of Machine Learning to Automated Analysis of Cerebral Edema in Large Cohorts of Ischemic Stroke Patients. Front. Neurol. 2018, 9, 687. [CrossRef]

68. Guberina, N.; Dietrich, U.; Radbruch, A.; Goebel, J.; Deuschl, C.; Ringelstein, A.; Köhrmann, M.; Kleinschnitz, C.; Forsting, M.; Mönninghoff, C. Detection of early infarction signs with machine learning-based diagnosis by means of the Alberta Stroke Program Early CT score (ASPECTS) in the clinical routine. Neuroradiology 2018, 60, 889-901. [CrossRef]

69. Fuchigami, T.; Akahori, S.; Okatani, T.; Li, Y. A hyperacute stroke segmentation method using 3D UNet integrated with physicians' knowledge for NCCT. Proc. SPIE 2020, 11314, 113140G.

70. Carey, L.M.; Seitz, R.J.; Parsons, M.; Levi, C.; Farquharson, S.; Tournier, J.-D.; Palmer, S.; Connelly, A. Beyond the lesion: Neuroimaging foundations for post-stroke recovery. Future Neurol. 2013, 8, 507-527. [CrossRef] 
71. Hirano, T. Searching for Salvageable Brain: The Detection of Ischemic Penumbra Using Various Imaging Modalities? J. Stroke Cerebrovasc. Dis. 2014, 23, 795-798. [CrossRef]

72. Maier, O.; Schröder, C.; Forkert, N.D.; Martinetz, T.; Handels, H. Correction: Classifiers for Ischemic Stroke Lesion Segmentation: A Comparison Study. PLoS ONE 2016, 11, e0149828. [CrossRef]

73. Mitra, J.; Bourgeat, P.; Fripp, J.; Ghose, S.; Rose, S.; Salvado, O.; Connelly, A.; Campbell, B.; Palmer, S.; Sharma, G.; et al. Lesion segmentation from multimodal MRI using random forest following ischemic stroke. NeuroImage 2014, 98, 324-335. [CrossRef]

74. Bharathi, P.G.; Agrawal, A.; Sundaram, P.; Sardesai, S.; Praveen, G. Combination of hand-crafted and unsupervised learned features for ischemic stroke lesion detection from Magnetic Resonance Images. Biocybern. Biomed. Eng. 2019, 39, 410-425. [CrossRef]

75. Yoo, A.J.; Barak, E.R.; Copen, W.A.; Kamalian, S.; Gharai, L.R.; Pervez, M.A.; Schwamm, L.H.; González, R.G.; Schaefer, P.W. Combining Acute Diffusion-Weighted Imaging and Mean Transmit Time Lesion Volumes with National Institutes of Health Stroke Scale Score Improves the Prediction of Acute Stroke. Stroke 2010, 41, 1728-1735. [CrossRef]

76. Maier, O.; Wilms, M.; von der Gablentz, J.; Krämer, U.M.; Münte, T.F.; Handels, H. Extra Tree forests for sub-acute ischemic stroke lesion segmentation in MR sequences. J. Neurosci. Methods 2015, 240, 89-100. [CrossRef]

77. Bouts, M.; Tiebosch, I.A.C.W.; Van Der Toorn, A.; Viergever, A.M.; Wu, O.; Dijkhuizen, R.M. Early Identification of Potentially Salvageable Tissue with MRI-Based Predictive Algorithms after Experimental Ischemic Stroke. Br. J. Pharmacol. 2013, 33, 1075-1082. [CrossRef]

78. Muschelli, J. Prediction of ischemic lesions using local image properties and random forests. In Proceedings of the Ischemic Stroke Lesion Segmentation (ISLES) challenge-MICCAI, Munich, Germany, 5 October 2015; pp. $63-68$.

79. Mahmood, Q.; Basit, A. Automatic Ischemic Stroke Lesion Segmentation in Multi-spectral MRI Images Using Random Forests Classifier. In BrainLes 2015, Proceedings of the First International Workshop, Held in Conjunction with MICCAI 2015, Munich, Germany, 5 October 2015; Lecture Notes in Computer Science; Springer: Cham, Switzerland, 2015; pp. 266-274.

80. Jesson, A.; Arbel, T. Hierarchical segmentation of normal and lesional structures combining an ensemble of probabilistic local classifiers and regional random forest classification. In Proceedings of the Ischemic Stroke Lesion Segmentation (ISLES) challenge-MICCAI, Munich, Germany, 5 October 2015; pp. 57-62.

81. Halme, H.-L.; Korvenoja, A.; Salli, E. ISLES (SISS) Challenge 2015: Segmentation of Stroke Lesions Using Spatial Normalization, Random Forest Classification and Contextual Clustering. In BrainLes 2015, Proceedings of the First International Workshop, Held in Conjunction with MICCAI 2015, Munich, Germany, 5 October 2015; Lecture Notes in Computer Science; Springer: Berlin/Heidelberg, Germany, 2016; pp. 211-221.

82. Jerman, T.; Galimzianova, A.; Pernuš, F.; Likar, B.; Špiclin, Ž. Combining Unsupervised and Supervised Methods for Lesion Segmentation. In BrainLes 2015, Proceedings of the First International Workshop, Held in Conjunction with MICCAI 2015, Munich, Germany, 5 October 2015; Lecture Notes in Computer Science; Springer: Berlin/Heidelberg, Germany, 2016; pp. 45-56.

83. McKinley, R.; Häni, L.; Wiest, R.; Reyes, M. Segmenting the Ischemic Penumbra: A Decision Forest Approach with Automatic Threshold Finding. In BrainLes 2015, Proceedings of the First International Workshop, Held in Conjunction with MICCAI 2015, Munich, Germany, 5 October 2015; Springer: Cham, Switzerland, 2015; pp. 275-283. [CrossRef]

84. Robben, D.; Christiaens, D.; Rangarajan, J.R.; Gelderblom, J.; Joris, P.; Maes, F.; Suetens, P. A Voxel-Wise, Cascaded Classification Approach to Ischemic Stroke Lesion Segmentation. In BrainLes 2015, Proceedings of the First International Workshop, Held in Conjunction with MICCAI 2015, Munich, Germany, 5 October 2015; Lecture Notes in Computer Science; Springer: Cham, Switzerland, 2015; Volume 9556, pp. 254-265.

85. Chen, G.; Li, Q.; Shi, F.; Rekik, I.; Pan, Z. RFDCR: Automated brain lesion segmentation using cascaded random forests with dense conditional random fields. NeuroImage 2020, 211, 116620. [CrossRef]

86. Goetz, M.; Weber, C.; Kolb, C.; Maier-Hein, K. Input Data Adaptive Learning (IDAL) for Sub-acute Ischemic Stroke Lesion Segmentation. In BrainLes 2015, Proceedings of the First International Workshop, Held in Conjunction with MICCAI 2015, Munich, Germany, 5 October 2015; Lecture Notes in Computer Science; Springer: Berlin/Heidelberg, Germany, 2016; Volume 9556, pp. $284-295$.

87. Griffanti, L.; Zamboni, G.; Khan, A.; Li, L.; Bonifacio, G.; Sundaresan, V.; Schulz, U.G.; Kuker, W.; Battaglini, M.; Rothwell, P.M.; et al. BIANCA (Brain Intensity AbNormality Classification Algorithm): A new tool for automated segmentation of white matter hyperintensities. NeuroImage 2016, 141, 191-205. [CrossRef]

88. Griffis, J.C.; Allendorfer, J.B.; Szaflarski, J.P. Voxel-based Gaussian naïve Bayes classification of ischemic stroke lesions in individual T1-weighted MRI scans. J. Neurosci. Methods 2016, 257, 97-108. [CrossRef]

89. Karthik, R.; Menaka, R. A multi-scale approach for detection of ischemic stroke from brain MR images using discrete curvelet transformation. Measurement 2017, 100, 223-232. [CrossRef]

90. Pereira, S.; Meier, R.; McKinley, R.; Wiest, R.; Alves, V.; Silva, C.A.; Reyes, M. Enhancing interpretability of automatically extracted machine learning features: Application to a RBM-Random Forest system on brain lesion segmentation. Med. Image Anal. 2018, 44, 228-244. [CrossRef]

91. Lin, C.-H.; Hsu, K.-C.; Johnson, K.R.; Luby, M.; Fann, Y.C. Applying density-based outlier identifications using multiple datasets for validation of stroke clinical outcomes. Int. J. Med. Inform. 2019, 132, 103988. [CrossRef]

92. Subudhi, A.K.; Acharya, U.R.; Dash, M.; Jena, S.; Sabut, S. Automated approach for detection of ischemic stroke using Delaunay Triangulation in brain MRI images. Comput. Biol. Med. 2018, 103, 116-129. [CrossRef] 
93. Peixoto, S.A.; Filho, P.P.R. Neurologist-level classification of stroke using a Structural Co-Occurrence Matrix based on the frequency domain. Comput. Electr. Eng. 2018, 71, 398-407. [CrossRef]

94. Garg, R.; Oh, E.; Naidech, A.; Kording, K.; Prabhakaran, S. Automating Ischemic Stroke Subtype Classification Using Machine Learning and Natural Language Processing. J. Stroke Cerebrovasc. Dis. 2019, 28, 2045-2051. [CrossRef]

95. Chen, H.; Khan, S.; Kou, B.; Nazir, S.; Liu, W.; Hussain, A. A Smart Machine Learning Model for the Detection of Brain Hemorrhage Diagnosis Based Internet of Things in Smart Cities. Complexity 2020, 2020, 3047869. [CrossRef]

96. Gillebert, C.; Humphreys, G.W.; Mantini, D. Automated delineation of stroke lesions using brain CT images. NeuroImage Clin. 2014, 4, 540-548. [CrossRef]

97. Bentley, P.; Ganesalingam, J.; Jones, A.L.C.; Mahady, K.; Epton, S.; Rinne, P.; Sharma, P.; Halse, O.; Mehta, A.; Rueckert, D. Prediction of stroke thrombolysis outcome using CT brain machine learning. NeuroImage Clin. 2014, 4, 635-640. [CrossRef]

98. Chin, C.-L.; Lin, B.-J.; Wu, G.-R.; Weng, T.-C.; Yang, C.-S.; Su, R.-C.; Pan, Y.-J. An automated early ischemic stroke detection system using CNN deep learning algorithm. In Proceedings of the 2017 IEEE 8th International Conference on Awareness Science and Technology (iCAST), Taichung, Taiwan, 8 November 2017; pp. 368-372.

99. Lisowska, A.; O’Neil, A.; Dilys, V.; Daykin, M.; Beveridge, E.; Muir, K.; Mclaughlin, S.; Poole, I. Context-Aware Convolutional Neural Networks for Stroke Sign Detection in Non-contrast CT Scans. In Proceedings of the Annual Conference on Medical Image Understanding and Analysis, 21st Annual Conference, MIUA 2017, Edinburgh, UK, 11-13 July 2017; Communications in Computer and Information Science; Springer: Berlin/Heidelberg, Germany, 2017; Volume 723, pp. $494-505$.

100. Abulnaga, S.M.; Rubin, J. Ischemic Stroke Lesion Segmentation in CT Perfusion Scans using Pyramid Pooling and Focal Loss. arXiv 2018, arXiv:1811.01085.

101. Lucas, C.; Kemmling, A.; Bouteldja, N.; Aulmann, L.F.; Mamlouk, A.M.; Heinrich, M.P. Learning to Predict Ischemic Stroke Growth on Acute CT Perfusion Data by Interpolating Low-Dimensional Shape Representations. Front. Neurol. 2018, 9, 989. [CrossRef]

102. Vargas, J.; Spiotta, A.; Chatterjee, A.R. Initial Experiences with Artificial Neural Networks in the Detection of Computed Tomography Perfusion Deficits. World Neurosurg. 2018, 124, e10-e16. [CrossRef]

103. Barman, A.; Inam, M.E.; Lee, S.; Savitz, S.; Sheth, S.; Giancardo, L. Determining Ischemic Stroke from CT-Angiography Imaging Using Symmetry-Sensitive Convolutional Networks. In Proceedings of the 2019 IEEE 16th International Symposium on Biomedical Imaging (ISBI 2019), Taichung, Taiwan, 8-10 November 2019; pp. 1873-1877.

104. Clèrigues, A.; Valverde, S.; Bernal, J.; Freixenet, J.; Oliver, A.; Llado, X. Acute ischemic stroke lesion core segmentation in CT perfusion images using fully convolutional neural networks. Comput. Biol. Med. 2019, 115, 103487. [CrossRef]

105. Shinohara, Y.; Takahashi, N.; Lee, Y.; Ohmura, T.; Kinoshita, T. Development of a deep learning model to identify hyperdense MCA sign in patients with acute ischemic stroke. Jpn. J. Radiol. 2020, 38, 112-117. [CrossRef] [PubMed]

106. Barros, S.R.; Tolhuisen, M.L.; Boers, A.M.; Jansen, I.; Ponomareva, E.; Dippel, D.W.J.; Lught, A.V.D.; Oostenbrugge, R.J.V.; Zwam, W.H.V.; Berkhemer, O.A.; et al. Automatic segmentation of cerebral infarcts in follow-up computed tomography images with convolutional neural networks. J. NeuroInterv. Surg. 2020, 12, 848-852. [CrossRef] [PubMed]

107. Öman, O.; Mäkelä, T.; Salli, E.; Savolainen, S.; Kangasniemi, M. 3D convolutional neural networks applied to CT angiography in the detection of acute ischemic stroke. Eur. Radiol. Exp. 2019, 3, 8. [CrossRef] [PubMed]

108. Hu, X.; Luo, W.; Hu, J.; Guo, S.; Huang, W.; Scott, M.R.; Wiest, R.; Dahlweid, M.; Reyes, M. Brain SegNet: 3D local refinement network for brain lesion segmentation. BMC Med. Imaging 2020, 20, 17. [CrossRef] [PubMed]

109. Islam, M.; Vaidyanathan, N.R.; Jose, V.J.M.; Ren, H. Ischemic Stroke Lesion Segmentation Using Adversarial Learning. Fundam. Softw. Eng. 2019, 11383, 292-300. [CrossRef]

110. Bertels, J.; Robben, D.; Vandermeulen, D.; Suetens, P. Contra-Lateral Information CNN for Core Lesion Segmentation Based on Native CTP in Acute Stroke. In Proceedings of the International MICCAI Brainlesion Workshop, 4th International Workshop, BrainLes 2018, Held in Conjunction with MICCAI 2018, Granada, Spain, 16 September 2018; Lecture Notes in Computer Science; Springer: Cham, Switzerland, 2019; pp. 263-270. [CrossRef]

111. Kuang, H.; Menon, B.K.; Sohn, S.I.; Qiu, W. EIS-Net: Segmenting early infarct and scoring ASPECTS simultaneously on non-contrast CT of patients with acute ischemic stroke. Med. Image Anal. 2021, 70, 101984. [CrossRef]

112. Avetisian, M.; Kokh, V.; Tuzhilin, A.; Umerenkov, D. Radiologist-level stroke classification on non-contrast CT scans with Deep U-Net. arXiv 2020, arXiv:2003.14287. [CrossRef]

113. Robben, D.; Boers, A.M.; Marquering, H.; Langezaal, L.L.; Roos, Y.B.; van Oostenbrugge, R.J.; van Zwam, W.H.; Dippel, D.W.; Majoie, C.B.; van der Lugt, A.; et al. Prediction of final infarct volume from native CT perfusion and treatment parameters using deep learning. Med. Image Anal. 2020, 59, 101589. [CrossRef]

114. Wang, C.-W.; Lee, J.-H. Stroke Lesion Segmentation of 3D Brain MRI Using Multiple Random Forests and 3D Registration. Adv. Data Min. Appl. 2016, 9556, 222-232.

115. Long, J.; Shelhamer, E.; Darrell, T. Fully convolutional networks for semantic segmentation. In Proceedings of the IEEE Conference on Computer Vision and Pattern Recognition, Boston, MA, USA, 7-12 June 2015; pp. 3431-3440. [CrossRef]

116. Havaei, M.; Dutil, F.; Pal, C.; Larochelle, H.; Jodoin, P.-M. A Convolutional Neural Network Approach to Brain Tumor Segmentation. In Brainlesion: Glioma, Multiple Sclerosis, Stroke and Traumatic Brain Injuries; Springer: Berlin/Heidelberg, Germany, 2016; Volume 9556, pp. 195-208. 
117. Stier, N.; Vincent, N.; Liebeskind, D.; Scalzo, F. Deep learning of tissue fate features in acute ischemic stroke. In Proceedings of the 2015 IEEE International Conference on Bioinformatics and Biomedicine (BIBM), Washington, DC, USA, 9-12 November 2015; Volume 2015, pp. 1316-1321.

118. Dou, Q.; Chen, H.; Yu, L.; Zhao, L.; Qin, J.; Wang, D.; Mok, V.; Shi, L.; Heng, P.A. Automatic Detection of Cerebral Microbleeds From MR Images via 3D Convolutional Neural Networks. IEEE Trans. Med. Imaging 2016, 35, 1182-1195. [CrossRef]

119. Choi, Y.; Kwon, Y.; Lee, H.; Kim, B.J.; Paik, M.C.; Won, J.-H. Ensemble of Deep Convolutional Neural Networks for Prognosis of Ischemic Stroke. In Computer Vision; Springer: Berlin/Heidelberg, Germany, 2016; Volume 10154, pp. $231-243$.

120. Wang, G.; Song, T.; Dong, Q.; Cui, M.; Huang, N.; Zhang, S. Automatic ischemic stroke lesion segmentation from computed tomography perfusion images by image synthesis and attention-based deep neural networks. Med. Image Anal. 2020, 65, 101787. [CrossRef]

121. Chen, L.; Bentley, P.; Rueckert, D. Fully automatic acute ischemic lesion segmentation in DWI using convolutional neural networks. NeuroImage Clin. 2017, 15, 633-643. [CrossRef]

122. Lucas, C.; Maier, O.; Heinrich, M.P. Shallow Fully-Connected Neural Networks for Ischemic Stroke-Lesion Segmentation in MRI In Informatik Aktuell; Springer: Berlin/Heidelberg, Germany, 2017; pp. 261-266.

123. Alex, V.; Vaidhya, K.; Thirunavukkarasu, S.; Kesavadas, C.; Krishnamurthi, G. Semi-supervised Learning using Denoising Autoencoders for Brain Lesion Detection and Segmentation. J. Med. Imaging 2017, 4, 041311. [CrossRef]

124. Giacalone, M.; Rasti, P.; Debs, N.; Frindel, C.; Cho, T.-H.; Grenier, E.; Rousseau, D. Local spatio-temporal encoding of raw perfusion MRI for the prediction of final lesion in stroke. Med. Image Anal. 2018, 50, 117-126. [CrossRef]

125. Lucas, C.; Kemmling, A.; Mamlouk, A.M.; Heinrich, M.P. Multi-scale neural network for automatic segmentation of ischemic strokes on acute perfusion images. In Proceedings of the 2018 IEEE 15th International Symposium on Biomedical Imaging (ISBI 2018), Washington, DC, USA, 4-7 April 2018; pp. 1118-1121.

126. Bento, M.; Souza, R.; Salluzzi, M.; Rittner, L.; Zhang, Y.; Frayne, R. Automatic identification of atherosclerosis subjects in a heterogeneous MR brain imaging data set. Magn. Reson. Imaging 2019, 62, 18-27. [CrossRef]

127. Song, T. Generative Model-Based Ischemic Stroke Lesion Segmentation. arXiv 2019, arXiv:1906.02392.

128. Liu, Z.; Cao, C.; Ding, S.; Han, T.; Liu, S. Towards Clinical Diagnosis: Automated Stroke Lesion Segmentation on Multi-Spectral MR Image Using Convolutional Neural Network. IEEE Access 2018, 6, 57006-57016. [CrossRef]

129. Zhang, R.; Zhao, L.; Lou, W.; Abrigo, J.; Mok, V.C.T.; Chu, W.C.W.; Wang, D.; Shi, L. Automatic Segmentation of Acute Ischemic Stroke From DWI Using 3-D Fully Convolutional DenseNets. IEEE Trans. Med. Imaging 2018, 37, 2149-2160. [CrossRef]

130. Chen, H.; Dou, Q.; Yu, L.; Qin, J.; Heng, P.A. VoxResNet: Deep voxelwise residual networks for brain segmentation from 3D MR images. NeuroImage 2018, 170, 446-455. [CrossRef]

131. Li, H.; Jiang, G.; Zhang, J.; Wang, R.; Wang, Z.; Zheng, W.-S.; Menze, B. Fully convolutional network ensembles for white matter hyperintensities segmentation in MR images. NeuroImage 2018, 183, 650-665. [CrossRef] [PubMed]

132. Praveen, G.; Agrawal, A.; Sundaram, P.; Sardesai, S. Ischemic stroke lesion segmentation using stacked sparse autoencoder. Comput. Biol. Med. 2018, 99, 38-52. [CrossRef] [PubMed]

133. Alex, V.; Safwan, K.P.M.; Chennamsetty, S.S.; Krishnamurthi, G. Generative adversarial networks for brain lesion detection. In Proceedings of the SPIE 10133, Medical Imaging 2017, Indian Institute of Technology, Madras, India, 24 February 2017. [CrossRef]

134. Li, H.; Zhygallo, A.; Menze, B. Automatic Brain Structures Segmentation Using Deep Residual Dilated U-Net. arXiv 2018, arXiv:1811.04312.

135. Luna, M.; Park, S.H. 3D Patchwise U-Net with Transition Layers for MR Brain Segmentation. In Proceedings of the International MICCAI Brainlesion Workshop, 4th International Workshop, BrainLes 2018, Held in Conjunction with MICCAI 2018, Granada, Spain, 16 September 2018; Springer: Cham, Switzerland, 2019; pp. 394-403. [CrossRef]

136. Winzeck, S.; Mocking, S.; Bezerra, R.; Bouts, M.; McIntosh, E.; Diwan, I.; Garg, P.; Chutinet, A.; Kimberly, W.; Copen, W.; et al. Ensemble of Convolutional Neural Networks Improves Automated Segmentation of Acute Ischemic Lesions Using Multiparametric Diffusion-Weighted MRI. Am. J. Neuroradiol. 2019, 40, 938-945. [CrossRef] [PubMed]

137. Liu, L.; Chen, S.; Zhang, F.; Wu, F.-X.; Pan, Y.; Wang, J. Deep convolutional neural network for automatically segmenting acute ischemic stroke lesion in multi-modality MRI. Neural Comput. Appl. 2020, 32, 6545-6558. [CrossRef]

138. Karthik, R.; Gupta, U.; Jha, A.; Rajalakshmi, R.; Menaka, R. A deep supervised approach for ischemic lesion segmentation from multimodal MRI using Fully Convolutional Network. Appl. Soft Comput. 2019, 84, 105685. [CrossRef]

139. Li, H.; Li, A.; Wang, M. A novel end-to-end brain tumor segmentation method using improved fully convolutional networks. Comput. Biol. Med. 2019, 108, 150-160. [CrossRef]

140. Malla, C.U.P.; Hernández, M.D.C.V.; Rachmadi, M.F.; Komura, T. Evaluation of Enhanced Learning Techniques for Segmenting Ischaemic Stroke Lesions in Brain Magnetic Resonance Perfusion Images Using a Convolutional Neural Network Scheme. Front. Aging Neurosci. 2019, 13, 33. [CrossRef]

141. Yang, H.; Huang, W.; Qi, K.; Li, C.; Liu, X.; Wang, M.; Zheng, H.; Wang, S. CLCI-Net: Crosslevel fusion and context inference networks for lesion segmentation of chronic stroke. In Proceedings of the International Conference on Medical Image Computing and Computer-Assisted Intervention, Shenzhen, China, 13-17 October 2019; pp. 266-274.

142. Qi, K.; Yang, H.; Li, C.; Liu, Z.; Wang, M.; Liu, Q.; Wang, S. X-net: Brain stroke lesion segmentation based on depthwise separable convolution and long-range dependencies. In Proceedings of the International Conference on Medical Image Computing and Computer-Assisted Intervention, Shenzhen, China, 13-17 October 2019; pp. 247-255. [CrossRef] 
143. Liu, L.; Wu, F.-X.; Wang, J. Efficient multi-kernel DCNN with pixel dropout for stroke MRI segmentation. Neurocomputing 2019, 350, 117-127. [CrossRef]

144. Chin, D.; Roderick, W.; Wang, K.M. Using Cascaded Networks for Post-Stroke Lesion Detection in the ATLAS Dataset. Available online: http:/ / cs230.stanford.edu/projects_spring_2018/reports/8288136.pdf (accessed on 4 October 2021).

145. Wang, Y.-R.; Wang, H.; Chen, S.; Katsaggelos, A.K.; Martersteck, A.; Higgins, J.; Hill, V.B.; Parrish, T. A 3D Cross-Hemisphere Neighborhood Difference Convnet for Chronic Stroke Lesion Segmentation. In Proceedings of the 2019 IEEE International Conference on Image Processing (ICIP), Taipei, Taiwan, 22-25 September 2019; pp. 1545-1549.

146. Wang, Y.; Katsaggelos, A.K.; Wang, X.; Parrish, T.B. A deep symmetry convnet for stroke lesion segmentation. In Proceedings of the 2016 IEEE International Conference on Image Processing (ICIP), Phoenix, AZ, USA, 3 August 2016; pp. 111-115.

147. Rajan, R.; Sathish, R.; Sheet, D. Significance of Residual Learning and Boundary Weighted Loss in Ischaemic Stroke Lesion Segmentation. arXiv 2019, arXiv:1908.04840.

148. Liu, L.; Kurgan, L.; Wu, F.-X.; Wang, J. Attention convolutional neural network for accurate segmentation and quantification of lesions in ischemic stroke disease. Med. Image Anal. 2020, 65, 101791. [CrossRef]

149. Zhang, L.; Song, R.; Wang, Y.; Zhu, C.; Liu, J.; Yang, J.; Liu, L. Ischemic Stroke Lesion Segmentation Using Multi-Plane Information Fusion. IEEE Access 2020, 8, 45715-45725. [CrossRef]

150. Amin, J.; Sharif, M.; Gul, N.; Raza, M.; Anjum, M.A.; Nisar, M.W.; Bukhari, S.A.C. Brain Tumor Detection by Using Stacked Autoencoders in Deep Learning. J. Med. Syst. 2019, 44, 32. [CrossRef]

151. Bui, T.D.; Ahn, S.; Lee, Y.; Shin, J. A Skip-Connected 3D DenseNet Networks with Adversarial Training for Volumetric Segmentation. In International MICCAI Brainlesion Workshop, Proceedings of the 4th International Workshop, BrainLes 2018, Held in Conjunction with MICCAI 2018, Granada, Spain, 16 September 2018; Springer: Cham, Switzerland, 2018; pp. 378-384. [CrossRef]

152. Joshi, S.; Gore, S. Ishemic Stroke Lesion Segmentation by Analyzing MRI Images Using Dilated and Transposed Convolutions in Convolutional Neural Networks. In Proceedings of the 2018 Fourth International Conference on Computing Communication Control and Automation (ICCUBEA), Pune, India, 16-18 August 2018; pp. 1-5.

153. Gupta, A.; Vupputuri, A.; Ghosh, N. Delineation of Ischemic Core and Penumbra Volumes from MRI using MSNet Architecture. In Proceedings of the 2019 41st Annual International Conference of the IEEE Engineering in Medicine and Biology Society (EMBC), Berlin, Germany, 23-27 July 2019; Volume 2019, pp. 6730-6733.

154. Kumar, A.; Upadhyay, N.; Ghosal, P.; Chowdhury, T.; Das, D.; Mukherjee, A.; Nandi, D. CSNet: A new DeepNet framework for ischemic stroke lesion segmentation. Comput. Methods Programs Biomed. 2020, 193, 105524. [CrossRef]

155. Sathish, R.; Rajan, R.; Vupputuri, A.; Ghosh, N.; Sheet, D. Adversarially Trained Convolutional Neural Networks for Semantic Segmentation of Ischaemic Stroke Lesion using Multisequence Magnetic Resonance Imaging. In Proceedings of the 2019 41st Annual International Conference of the IEEE Engineering in Medicine and Biology Society (EMBC), Berlin, Germany, 23-27 July 2019; Volume 2019, pp. 1010-1013.

156. Phong, T.D.; Duong, H.N.; Nguyen, H.T.; Trong, N.T.; Nguyen, V.H.; Van Hoa, T.; Snasel, V. Brain Hemorrhage Diagnosis by Using Deep Learning. In Proceedings of the 2017 International Conference on Machine Learning and Soft Computing, Ho Chi Minh City, Vietnam, 13-16 January 2017; ACM Press: New York, NY, USA, 2017; pp. 34-39.

157. Majumdar, A.; Brattain, L.; Telfer, B.; Farris, C.; Scalera, J. Detecting Intracranial Hemorrhage with Deep Learning. In Proceedings of the 2018 40th Annual International Conference of the IEEE Engineering in Medicine and Biology Society (EMBC), Honolulu, HI, USA, 17-21 July 2018; Volume 2018, pp. 583-587.

158. Arbabshirani, M.R.; Fornwalt, B.K.; Mongelluzzo, G.J.; Suever, J.D.; Geise, B.D.; Patel, A.A.; Moore, G.J. Advanced machine learning in action: Identification of intracranial hemorrhage on computed tomography scans of the head with clinical workflow integration. NPJ Digit. Med. 2018, 1, 9. [CrossRef]

159. Kuo, W.; Häne, C.; Mukherjee, P.; Malik, J.; Yuh, E.L. Expert-level detection of acute intracranial hemorrhage on head computed tomography using deep learning. Proc. Natl. Acad. Sci. USA 2019, 116, 22737-22745. [CrossRef]

160. Patel, A.; Schreuder, F.H.B.M.; Klijn, C.J.M.; Prokop, M.; Van Ginneken, B.; Marquering, H.; Roos, Y.B.W.E.M.; Baharoglu, M.I.; Meijer, F.J.A.; Manniesing, R. Intracerebral Haemorrhage Segmentation in Non-Contrast CT. Sci. Rep. 2019, 9, 17858. [CrossRef]

161. Cho, J.; Park, K.-S.; Karki, M.; Lee, E.; Ko, S.; Kim, J.K.; Lee, D.E.; Choe, J.; Son, J.; Kim, M.; et al. Improving Sensitivity on Identification and Delineation of Intracranial Hemorrhage Lesion Using Cascaded Deep Learning Models. J. Digit. Imaging 2019, 32, 450-461. [CrossRef]

162. Patel, A.; van de Leemput, S.C.; Prokop, M.; Van Ginneken, B.; Manniesing, R. Image Level Training and Prediction: Intracranial Hemorrhage Identification in 3D Non-Contrast CT. IEEE Access 2019, 7, 92355-92364. [CrossRef]

163. Barros, R.S.; Van Der Steen, W.E.; Boers, A.M.; Zijlstra, I.; Berg, R.V.D.; El Youssoufi, W.; Urwald, A.; Verbaan, D.; Vandertop, P.; Majoie, C.; et al. Automated segmentation of subarachnoid hemorrhages with convolutional neural networks. Inform. Med. Unlocked 2020, 19, 100321. [CrossRef]

164. Lee, J.Y.; Kim, J.S.; Kim, T.Y.; Kim, Y.S. Detection and classification of intracranial haemorrhage on CT images using a novel deep-learning algorithm. Sci. Rep. 2020, 10, 20546. [CrossRef] [PubMed]

165. Xu, Y.; Holanda, G.; Souza, L.F.d.F.; Silva, H.; Gomes, A.; Silva, I.; Ferreira, M.; Jia, C.; Han, T.; de Albuquerque, V.H.C.; et al. Deep Learning-Enhanced Internet of Medical Things to Analyze Brain CT Scans of Hemorrhagic Stroke Patients: A New Approach IEEE Sens. J. 2020, 21, 24941-24951. [CrossRef] 
166. Li, L.; Wei, M.; Liu, B.; Atchaneeyasakul, K.; Zhou, F.; Pan, Z.; Kumar, S.A.; Zhang, J.Y.; Pu, Y.; Liebeskind, D.S.; et al. Deep Learning for Hemorrhagic Lesion Detection and Segmentation on Brain CT Images. IEEE J. Biomed. Health Inform. 2021, 25, 1646-1659. [CrossRef] [PubMed]

167. Arab, A.; Chinda, B.; Medvedev, G.; Siu, W.; Guo, H.; Gu, T.; Moreno, S.; Hamarneh, G.; Ester, M.; Song, X. A fast and fullyautomated deep-learning approach for accurate hemorrhage segmentation and volume quantification in non-contrast whole-head CT. Sci. Rep. 2020, 10, 19389. [CrossRef]

168. Grewal, M.; Srivastava, M.M.; Kumar, P.; Varadarajan, S. RADnet: Radiologist level accuracy using deep learning for hemorrhage detection in CT scans. In Proceedings of the 2018 IEEE 15th International Symposium on Biomedical Imaging (ISBI 2018), Washington, DC, USA, 4-7 April 2018; pp. 281-284.

169. Burduja, M.; Ionescu, R.; Verga, N. Accurate and Efficient Intracranial Hemorrhage Detection and Subtype Classification in 3D CT Scans with Convolutional and Long Short-Term Memory Neural Networks. Sensors 2020, 20, 5611. [CrossRef]

170. Pereira, D.R.; Filho, P.P.R.; de Rosa, G.H.; Papa, J.P.; de Albuquerque, V.H.C. Stroke Lesion Detection Using Convolutional Neural Networks. In Proceedings of the 2018 International Joint Conference on Neural Networks (IJCNN), Rio de Janeiro, Brazil, 8-13 July2018; pp. 1-6.

171. Marbun, J.T.; Seniman; Andayani, U. Classification of stroke disease using convolutional neural network. J. Phys. Conf. Ser. 2018, 978, 012092. [CrossRef]

172. Dourado, C.M.J.M., Jr.; da Silva, S.P.P.; da Nóbrega, R.V.M.; Barros, A.C.D.S.; Filho, P.P.R.; de Albuquerque, V.H.C. Deep learning IoT system for online stroke detection in skull computed tomography images. Comput. Netw. 2019, 152, 25-39. [CrossRef]

173. Kuang, H.; Menon, B.K.; Qiu, W. Segmenting Hemorrhagic and Ischemic Infarct Simultaneously from Follow-Up Non-Contrast CT Images in Patients with Acute Ischemic Stroke. IEEE Access 2019, 7, 39842-39851. [CrossRef]

174. Xue, Y.; Farhat, F.G.; Boukrina, O.; Barrett, A.; Binder, J.R.; Roshan, U.W.; Graves, W.W. A multi-path 2.5 dimensional convolutional neural network system for segmenting stroke lesions in brain MRI images. NeuroImage Clin. 2020, 25, 102118. [CrossRef]

175. Bria, A.; Marrocco, C.; Tortorella, F. Addressing class imbalance in deep learning for small lesion detection on medical images. Comput. Biol. Med. 2020, 120, 10373. [CrossRef]

176. Sipser, M. Introduction to the Theory of Computation. ACM Sigact News 1996, 27, 27-29. [CrossRef]

177. Huang, K.; Rhee, D.J.; Ger, R.; Layman, R.; Yang, J.; Cardenas, C.E.; Court, L.E. Impact of slice thickness, pixel size, and CT dose on the performance of automatic contouring algorithms. J. Appl. Clin. Med. Phys. 2021, 22, 168-174. [CrossRef] 